\title{
An exceptional collection for Khovanov homology
}

\author{
BENJAMIN COOPER \\ MATT HOGANCAMP
}

\begin{abstract}
The Temperley-Lieb algebra is a fundamental component of SU(2) topological quantum field theories. We construct chain complexes corresponding to minimal idempotents in the Temperley-Lieb algebra. Our results apply to the framework which determines Khovanov homology. Consequences of our work include semiorthogonal decompositions of categorifications of Temperley-Lieb algebras and Postnikov decompositions of all Khovanov tangle invariants.
\end{abstract}

57R56; 57M27

\section{Introduction}

The purpose of this paper is to give a general method for lifting an idempotent decomposition of the Temperley-Lieb algebra $\mathrm{TL}_{n}$ to a decomposition of its categorification. Roughly speaking, we lift each idempotent $p \in \mathrm{TL}_{n}$ to a chain complex $P \in \operatorname{Kom}(n)$ so that the equation

$$
p \cdot p=p \text { lifts to } P \otimes P \simeq P .
$$

Along the way, we find new skein-theoretic expressions for the decomposition of the Temperley-Lieb algebra, the categories which determine Khovanov homology are extended to differential graded categories, some mapping spaces are computed, and a complete decomposition of these categories is introduced.

\subsection{The Temperley-Lieb algebra and its decomposition}

A $k$-algebra $A$ can be often be expressed as a sum

$$
A=A_{1} \oplus A_{2} \oplus \cdots \oplus A_{N} .
$$

This information can be encoded by a collection of elements $\left\{p_{i}\right\}_{i=1}^{N}$ called projectors or idempotents. Each $p_{i} \in A$ determines a projection and an inclusion

$$
A \stackrel{p_{i} \cdot}{\longrightarrow} A_{i} \hookrightarrow A
$$


to and from the subspace $A_{i}$ in $A$. Equation (1-1) implies that the collection $\left\{p_{i}\right\}$ satisfies the equations

$$
p_{i} \cdot p_{i}=p_{i}, \quad p_{i} \cdot p_{j}=0 \text { when } i \neq j, \quad \text { and } \quad 1_{A}=\sum_{i=1}^{N} p_{i} .
$$

If each projector $p_{i}$ cannot be written as a sum of two non-trivial projectors then the collection $\left\{p_{i}\right\}$ is a complete set of primitive, mutually orthogonal idempotents.

Suppose that $\mathrm{U}_{q} \mathfrak{s l}(2)$ is the quantum group associated to the Lie algebra $\mathfrak{s l}(2)$ and $V=V_{1}$ is the 2-dimensional irreducible representation corresponding to the action of $\mathfrak{s l}(2)$ on $\mathbb{C}^{2}$. The Temperley-Lieb algebra $\mathrm{TL}_{n}$ is the endomorphism algebra of the $n$-fold tensor power of $V$ :

$$
\mathrm{TL}_{n}=\operatorname{End}_{\mathrm{U}_{q} \mathfrak{s l}(2)}\left(V^{\otimes n}\right) .
$$

The inner product $V \otimes V \rightarrow \mathbb{C}(q)$ and its dual $\mathbb{C}(q) \rightarrow V \otimes V$ generate the TemperleyLieb algebra $\mathrm{TL}_{n}$. Drawing the latter as a cup and the former as a cap gives rise to a pictorial representation of every element in $\mathrm{TL}_{n}$. For example, the composition

$$
V \otimes V \rightarrow \mathbb{C}(q) \rightarrow V \otimes V \quad \text { is pictured as } \bigcup_{\cap} \in \mathrm{TL}_{2} .
$$

This graphical interpretation leads to an alternative, topological, definition of the Temperley-Lieb algebras as quotients of categories of 1-dimensional cobordisms. An important consequence of these two different definitions is the ability to characterize constructions involving the Temperley-Lieb algebra in two very different ways.

The Temperley-Lieb algebra is semi-simple and a complete set of primitive mutually orthogonal idempotents can be described using representation theory. If $V_{k}$ is the $k^{\text {th }}$ irreducible representation of $\mathfrak{s l}(2)$ then the tensor product $V_{1}^{\otimes n}$ can be written as a direct sum of irreducible representations

$$
V_{1}^{\otimes n} \cong V_{n} \oplus m_{n-2} V_{n-2} \oplus m_{n-4} V_{n-4} \oplus \cdots .
$$

The number $m_{k}=\operatorname{dim}_{\mathbb{C}(q)} \operatorname{Hom}\left(V_{k}, V_{1}^{\otimes n}\right)$ is the multiplicity of $V_{k}$ in $V_{1}^{\otimes n}$. Corresponding to each summand $V_{k} \subset m_{k} V_{k} \subset V_{1}^{\otimes n}$ is an idempotent

$$
p: V_{1}^{\otimes n} \rightarrow V_{k} \hookrightarrow V_{1}^{\otimes n} \in \mathrm{TL}_{n} .
$$

For example, the multiplicity $m_{n}$ of $V_{n}$ in $V_{1}^{\otimes n}$ is always equal to 1 and the idempotent associated to $V_{n} \subset V_{1}^{\otimes n}$ is the famous Jones-Wenzl projector (see Wenzl [29]). In this paper, we are interested in the entire collection

$$
\left\{p_{W}: W \subset V_{1}^{\otimes n} \text { such that } W \cong V_{k} \text { for some } k \text { in (1-3) }\right\} .
$$


Unfortunately, the definition given above is not useful in practice. In Section 2, we will begin by finding more convenient expressions for these idempotents in terms of the Jones-Wenzl projectors.

Example 1.2 When $n=4$, there is a decomposition

$$
V_{1}^{\otimes 4}=V_{4} \oplus 3 V_{2} \oplus 2 V_{0},
$$

which contains a unique copy of the 5-dimensional irreducible representation $V_{4}$, three copies of the 3-dimensional representation $V_{2}$ and two copies of the 1-dimensional trivial representation $V_{0}$. There are six idempotents $p_{\epsilon}$ in the Temperley-Lieb algebra $\mathrm{TL}_{4}$; each corresponding to projecting onto distinct irreducible summands. They are pictured below:

$$
\begin{aligned}
& p_{4,4}=p_{(1,1,1,1)}=\frac{\uplus 1}{\pi},
\end{aligned}
$$

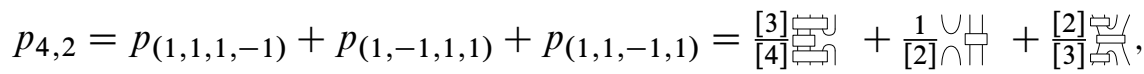

$$
\begin{aligned}
& p_{4,0}=p_{(1,-1,1,-1)}+p_{(1,1,-1,-1)}=\frac{1}{[2]^{2}} \cap \cup+\frac{1}{[3]} \cup
\end{aligned}
$$

In this illustration, the number $[n] \in \mathbb{Z}\left[q, q^{-1}\right]$ is the $n^{\text {th }}$ quantum integer

$$
[n]=q^{-(n-1)}+q^{-(n-3)}+\cdots+q^{n-3}+q^{n-1} .
$$

The subscript $\epsilon=\left(\epsilon_{1}, \ldots, \epsilon_{4}\right)$ indicates that $p_{\epsilon}$ corresponds to projection onto a distinct summand isomorphic to $V_{\epsilon_{1}+\cdots+\epsilon_{4}}$, see Definition 2.15. The $k^{\text {th }}$ row corresponds to the $k^{\text {th }}$ isotypic or higher-order projector $p_{n, k} \in \mathrm{TL}_{n}$. The boxes in the pictures above represent Jones-Wenzl projectors $p_{n} \in \mathrm{TL}_{n}$.

\subsection{Categorifications and decompositions}

The idea behind categorification is to replace a $k$-algebra $A$ by a monoidal category $\mathcal{A}$. When $\mathcal{A}$ is monoidal the Grothendieck group $\mathrm{K}_{0}(\mathcal{A})$ becomes a ring. A category $\mathcal{A}$ categorifies a $k$-algebra $A$ when there is an isomorphism

$$
\mathrm{K}_{0}(\mathcal{A}) \otimes_{\mathbb{Z}} k \stackrel{\sim}{\longrightarrow} A .
$$

There are as many examples of categorifications as there are monoidal categories for which the Grothendieck group functor can be defined. In order to ensure that something interesting happens one usually asks for the category $\mathcal{A}$ to satisfy some additional properties.

In [16], Khovanov introduced a categorification of the Jones polynomial. In subsequent papers [17;2], this homological invariant of links was refined to a local invariant of 
tangles, taking values in categories $\operatorname{Kom}(n)$. There are isomorphisms

$$
\mathrm{K}_{0}(\operatorname{Kom}(n)) \otimes_{\mathbb{Z}} \mathbb{C}(q) \stackrel{\sim}{\longrightarrow} \mathrm{TL}_{n},
$$

making the categories $\operatorname{Kom}(n)$ categorifications of the Temperley-Lieb algebras $\mathrm{TL}_{n}$. In addition to being categorifications, they satisfy the constraint that they determine knot invariants.

Other categorifications of the Temperley-Lieb algebra have been shown to lead to knot invariants. Some come from derived categories of coherent sheaves, others come from enumerative invariants of Lagrangian fibrations, perverse sheaves on Grassmannians, the category $\mathcal{O}$, matrix factorizations or sheaves concentrated on type $A_{2}$ singularities; see Cautis and Kamnitzer [6], Seidel and Smith [26], Stroppel [27], Bernstein, Frenkel and Khovanov [3], Khovanov and Rozansky [19], Wu [30], and Orlov [22]. However, the requirement that such categories determine knot invariants is very strong (see Khovanov [18]). The categories $\operatorname{Kom}(n)$ are minimal with respect to these constraints. This makes them the ideal setting for constructions which apply to other categorifications in this family.

If an algebra $A$ is categorified by $\mathcal{A}$ then an element $p \in A$ is categorified by a choice of $P \in \mathcal{A}$ for which $\mathrm{K}_{0}(P)=p$. Whenever one encounters an element $p$ in an algebra $A$, one can ask for lifts $P \in \mathcal{A}$. Equation (1-4) implies that there will be at least one lift, but there may be others depending upon how many extensions exist between objects in the category $\mathcal{A}$.

In Cooper and Krushkal [8], the Jones-Wenzl projector $p_{n} \in \mathrm{TL}_{n}$ was lifted to a chain complex $P_{n} \in \operatorname{Kom}(n)$ which satisfies $\mathrm{K}_{0}\left(P_{n}\right)=p_{n}$. In addition to being idempotent, $P_{n} \otimes P_{n} \simeq P_{n}$, the lift $P_{n}$ was characterized uniquely up to homotopy in the category $\operatorname{Kom}(n)$. The properties which determine $P_{n}$ are given in terms of the topological description of the category $\operatorname{Kom}(n)$ and provide a unique counterpoint to algebraic descriptions that can be obtained by Yoneda's Lemma or localization (as in Section 8.15). There are deep relations between the categorified projectors $P_{n}$ and constructions in mathematical physics and algebraic geometry; see Gorsky, Oblomkov, Rasmussen and Shende [12], Gukov and Stošić [13], and Rozansky [25].

One of the main results of this paper is the construction of chain complexes $P_{\epsilon} \in \operatorname{Kom}(n)$ corresponding to each of the idempotents $p_{\epsilon} \in \mathrm{TL}_{n}$ which were discussed in the previous section. More precisely, we show:

Theorem For each $\epsilon$, there is a chain complex $P_{\epsilon} \in \operatorname{Kom}(n)$ such that $\mathrm{K}_{0}\left(P_{\epsilon}\right)=p_{\epsilon}$.

Theorem The chain complexes $P_{\epsilon}$ are idempotent and mutually orthogonal:

$$
P_{\epsilon} \otimes P_{\epsilon} \simeq P_{e} \quad \text { and } \quad P_{\epsilon} \otimes P_{\delta} \simeq 0 \text { when } \epsilon \neq \delta
$$


Theorem The projectors $P_{\epsilon}$ glue together to form a chain complex $R_{n}$ which satisfies

$$
1_{n} \simeq R_{n},
$$

where $1_{n} \in \operatorname{Kom}(n)$ is the monoidal identity. This homotopy equivalence corresponds to the second part of Equation (1-2) above.

An important new ingredient in this setting is the maps between objects. In the Temperley-Lieb algebra, different subspaces did not interact because they corresponded to the images of distinct irreducible summands of $V_{1}^{\otimes n}$. After lifting the idempotents $p_{\epsilon}$ defining these subspaces to objects $P_{\epsilon} \in \operatorname{Kom}(n)$, we find that they must interact: there are non-trivial maps between idempotents. However, a more refined statement can be made. We address the question of what this interaction looks like in the theorem below:

Theorem The mapping spaces between projectors which do not respect the dominance order are contractible:

$$
\epsilon \not \unlhd \delta \text { implies } \operatorname{Hom}^{*}\left(P_{\epsilon}, P_{\delta}\right) \simeq 0
$$

see Section 5.

It follows from this theorem that all of the objects in the categories $\operatorname{Kom}(n)$ are filtered by the projectors $P_{\epsilon}$. In turn, this filtration can be used to define Postnikov towers for all objects, including tangle invariants. The idempotents $\left\{P_{\epsilon}\right\}$ form an exceptional collection for Khovanov homology.

Example 1.4 The picture of the resolution of identity $R_{4}$ captures many aspects of the information conveyed above. Each projector $p_{\epsilon}$ in Example 1.2 lifts to a chain complex $P_{\epsilon}$ that satisfies $\mathrm{K}_{0}\left(P_{\epsilon}\right)=p_{\epsilon}$. These categorified projectors form the vertices of the diagram below. The arrows represent non-trivial maps between projectors that control the decomposition of the category $\operatorname{Kom}(4)$. There are no arrows pointing from right to left:

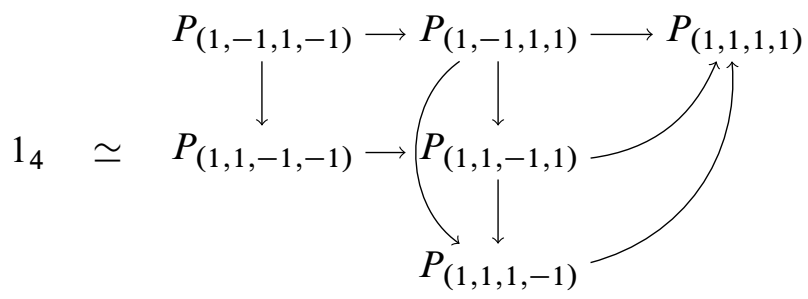




\section{The Temperley-Lieb category and higher-order projectors}

In this section we summarize basic information about the Temperley-Lieb category $\mathrm{TL}$, the Temperley-Lieb algebra $\mathrm{TL}_{n}$, and the Jones-Wenzl projectors $p_{n} \in \mathrm{TL}_{n}$. In Section 2.10, the higher-order projectors $p_{n, k} \in \mathrm{TL}_{n}$ are defined representation theoretically. In Section 2.14, new projectors $p_{\epsilon} \in \mathrm{TL}_{n}$ are introduced and related to the higher-order projectors. This allows us to characterize $p_{n, k}$ uniquely in terms of its interaction with other Temperley-Lieb elements. It is this latter definition which will lift to chain complexes in Section 8. For more information about the Temperley-Lieb algebra and its connection to low-dimensional topology, see [15].

\subsection{Temperley-Lieb category}

Here we define the Temperley-Lieb category TL and establish some basic notions, such as the through-degree $\tau(a)$ of elements $a \in \mathrm{TL}$.

Definition 2.2 The Temperley-Lieb category TL is the category of $\mathrm{U}_{q} \mathfrak{s l}(2)$-equivariant maps from $n$-fold to $k$-fold tensor powers of the fundamental representation $V$. More specifically, the objects of TL are indexed by integers $n$ corresponding to tensor powers $V^{\otimes n}$ of the fundamental representation, and the morphisms

$$
\operatorname{TL}(n, k)=\operatorname{Hom}_{\mathrm{U}_{q} \mathfrak{s l}(2)}\left(V^{\otimes n}, V^{\otimes k}\right)
$$

are determined by $\mathrm{U}_{q} \mathfrak{s l}(2)$-equivariant maps. The elements of $\operatorname{TL}(n, k)$ can be represented by $\mathbb{C}(q)$-linear combinations of pictures consisting of chords from a collection of $n$ points to a collection of $k$ points situated on two horizontal lines in the plane. Such pictures correspond to compositions of the maps

$$
\mathbb{C}(q) \rightarrow V \otimes V \quad \text { and } \quad V \otimes V \rightarrow \mathbb{C}(q) .
$$

Pictures are considered equivalent when they are isotopic relative to the boundary. We also impose the relation that a disjoint circle can be removed at the cost of multiplying by $q+q^{-1}$, the graded dimension of $V$.

A sample element of the space of morphisms from four points to six points is pictured below:

$$
\overbrace{\cap}^{\cup} \in \operatorname{TL}(4,6)
$$

When elements are represented by such pictures, the composition

$$
\mathrm{TL}(n, k) \otimes \mathrm{TL}(k, l) \rightarrow \mathrm{TL}(n, l), \quad \text { where } a \otimes b \mapsto b a
$$


in the Temperley-Lieb category corresponds to vertical stacking.

Definition 2.3 There are two operations relating different parts of TL that will be used repeatedly:

$$
x \mapsto x \sqcup 1 \quad \text { and } \quad x \mapsto \bar{x} .
$$

For each element $x \in \operatorname{TL}(n, k)$ there is an element $x \sqcup 1 \in \operatorname{TL}(n+1, k+1)$ obtained by placing a single vertical strand to the right of all of the diagrams appearing in the expression for $x$. Given an element $x \in \operatorname{TL}(n, k)$ there is a corresponding element $\bar{x} \in \operatorname{TL}(k, n)$ obtained by flipping the diagrams representing $x$ upside down. Both of these operations are $q$-linear.

Definition 2.4 The Temperley-Lieb algebra $\mathrm{TL}_{n}$ is given by the endomorphisms of the $n^{\text {th }}$ object in the Temperley-Lieb category,

$$
\mathrm{TL}_{n}=\mathrm{TL}(n, n)=\operatorname{End}_{\mathrm{U}_{q} \mathfrak{s l}(2)}\left(V^{\otimes n}\right) .
$$

Definition 2.5 The elements of $\mathrm{TL}_{n}$ are generated by elementary diagrams $e_{i}$ containing $n-2$ vertical chords and two horizontal chords connecting the $i^{\text {th }}$ and the $i+1^{\text {st }}$ positions. For instance,

$$
e_{1}=\bigcup \mid \in \mathrm{TL}_{3} .
$$

Definition 2.6 If $a \in \operatorname{TL}(n, m)$ is a Temperley-Lieb diagram then there are many ways in which $a$ factors as a composition $a=c b$ where $b \otimes c \in \operatorname{TL}(n, l) \otimes \operatorname{TL}(l, m)$. The through-degree $\tau(a)$ of $a$ is equal to the minimal $l$ achieved by such a factorization. If $a \in \operatorname{TL}(n, m)$ is a linear combination of Temperley-Lieb diagrams, $a=\sum_{i} f_{i} a_{i}$, then the through-degree of $a$ is defined by

$$
\tau(a)=\max _{i}\left\{\tau\left(a_{i}\right) \mid f_{i} \neq 0\right\} .
$$

Example 2.7 The through-degrees $\tau$ of the two diagrams (2-2) and $e_{1}$, pictured above, are two and one respectively.

Remark 2.8 Through-degree cannot increase when composing elements of TL. In this manner, the category TL is filtered by through-degree. Let $\mathrm{TL}^{k} \subset \mathrm{TL}$ denote the subcategory consisting of morphisms that have a through-degree which is less than $k$,

$$
\mathrm{TL}^{k}(n, m)=\{f \in \mathrm{TL}(n, m): \tau(f)<k\} .
$$


Then there is a filtration

$$
\cdots \subset \mathrm{TL}^{k-1} \subset \mathrm{TL}^{k} \subset \mathrm{TL}^{k+1} \subset \cdots \quad \text { and } \quad \mathrm{TL}=\bigcup_{k} \mathrm{TL}^{k}
$$

which is respected by operations in the sense that

$$
\mathrm{TL}^{k} \otimes \mathrm{TL}^{k} \stackrel{\circ}{\rightarrow} \mathrm{TL}^{k} \quad \text { and } \quad \mathrm{TL}^{k} \otimes \mathrm{TL}^{l} \stackrel{\sqcup}{\rightarrow} \mathrm{TL}^{k+l} .
$$

Remark 2.9 Instead of through-degree, one might choose instead to filter elements $a \in \mathrm{TL}_{n}$ by the number of turnbacks,

$$
\cap(a)=(n-\tau(a)) / 2 .
$$

This convention also appears in the literature. While Theorem 2.26 and Definition 8.4 can be stated in terms of turnbacks, it is awkward to use $\cap(a)$ for general elements of the category TL.

\subsection{Idempotents from irreducibles}

In this section we will explain the connection between $\mathrm{U}_{q} \mathfrak{s l}(2)$ representation theory and higher-order Jones-Wenzl projectors. These higher-order Jones-Wenzl projectors will be explored in Section 2.14 and categorified in Section 7.

The irreducible representations $V_{k}$ of $\mathrm{U}_{q} \mathfrak{s l}(2)$ are indexed by integers $k \in \mathbb{Z}_{\geq 0}$. The trivial representation $V_{0}$ is 1 -dimensional and the fundamental representation $V_{1} \cong V$ is 2-dimensional. In general, we can use the Clebsch-Gordan rule,

$$
V_{n} \otimes V_{1} \cong V_{n+1} \oplus V_{n-1},
$$

to decompose the tensor product $V^{\otimes n}$ into a direct sum of irreducible representations

$$
V^{\otimes n} \cong V_{n} \oplus m_{n-2} V_{n-2} \oplus m_{n-4} V_{n-4} \oplus \cdots .
$$

For each summand $m_{k} V_{k} \subset V^{\otimes n}$ there are equivariant projection and inclusion maps,

$$
V^{\otimes n} \stackrel{\pi_{n, k}}{\longrightarrow} m_{k} V_{k} \stackrel{i_{n, k}}{\longrightarrow} V^{\otimes n}
$$

and Equation (2-1) implies the existence of a Temperley-Lieb element $p_{n, k}=i_{n, k}$ 。 $\pi_{n, k} \in \mathrm{TL}_{n}$.

Definition 2.11 The $k^{\text {th }}$ higher-order Jones-Wenzl projector is the idempotent element $p_{n, k} \in \mathrm{TL}_{n}$ corresponding to the summand $m_{k} V_{k} \subset V^{\otimes n}$.

In the remainder of this section we will provide several descriptions of these idempotents. A different discussion can be found in [10;23]. 


\subsection{Jones-Wenzl projectors}

The Jones-Wenzl projectors $p_{n} \in \mathrm{TL}_{n}$ are a special case of Definition 2.11. The largest irreducible summand $V_{n} \subset V^{\otimes n}$ occurs with multiplicity one, $m_{n}=1$. Since the Jones-Wenzl projectors correspond to projection onto this summand,

$$
V^{\otimes n} \rightarrow V_{n} \rightarrow V^{\otimes n}
$$

they correspond to the higher-order Jones-Wenzl projector of largest degree, $p_{n}=p_{n, n}$. In [29], Wenzl introduced projectors using the recurrence relation

$$
p_{1}=1 \quad \text { and } \quad p_{n}=p_{n-1} \sqcup 1-\frac{[n-1]}{[n]}\left(p_{n-1} \sqcup 1\right) e_{n-1}\left(p_{n-1} \sqcup 1\right),
$$

where the quantum integer $[n]$ is defined to be the Laurent polynomial

$$
[n]=\frac{q^{n}-q^{-n}}{q-q^{-1}}=q^{-(n-1)}+q^{-(n-3)}+\cdots+q^{n-3}+q^{n-1} .
$$

If we depict $p_{n}$ graphically by a box with $n$ incoming and $n$ outgoing chords

$$
p_{n}=\underset{|n|}{|c|},
$$

then Equation (2-4) can be illustrated using diagrams:

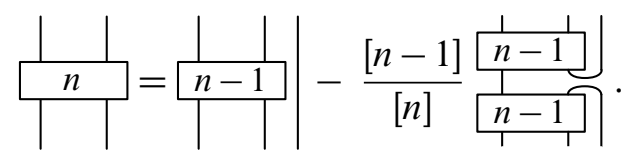

It can be shown that the Jones-Wenzl projectors are uniquely characterized by the following properties:

(1) $p_{n} \in \mathrm{TL}_{n}$.

(2) $p_{n}-1$ belongs to the subalgebra generated by $\left\{e_{1}, e_{2}, \ldots, e_{n-1}\right\}$.

(3) $e_{i} p_{n}=p_{n} e_{i}=0$ for all $i=1, \ldots, n-1$.

For more information see $[29 ; 15 ; 8]$.

Remark 2.13 Although the coefficient ring $\mathbb{C}(q)$ is used throughout Section 2, we will consistently interpret expressions like $[n] /[n+1]$ as a power series in the ring $\mathbb{Z}\left[q^{-1}\right] \llbracket q \rrbracket$. See $[8 ; 9]$ for discussion. 


\subsection{Higher-order Jones-Wenzl projectors}

Recall from Section 2.10 that the $\mathrm{U}_{q} \mathfrak{s l}(2)$ representation $V^{\otimes n}$ decomposes as a sum of irreducible representations $V_{k}$,

$$
V^{\otimes n} \cong \bigoplus_{k} m_{k} V_{k}
$$

Each summand $m_{k} V_{k} \subset V^{\otimes n}$ is a sum of $m_{k}$ copies of the $k^{\text {th }}$ irreducible representation $V_{k}$. For each higher-order projector $p_{n, k}$ we would like to write an equation of the form

$$
p_{n, k}=\sum_{\epsilon \in \mathcal{L}_{n, k}} p_{\epsilon}
$$

where $\mathcal{L}_{n, k}$ is a set indexing copies of $V_{k}$ in the summand $m_{k} V_{k} \subset V^{\otimes n}$ so that $\left|\mathcal{L}_{n, k}\right|=m_{k}$. Each element $p_{\epsilon} \in \mathrm{TL}_{n}$ corresponds to projection onto a distinct copy of $V_{k}$ within $m_{k} V_{k}$. This notation is introduced by the definition below.

Definition 2.15 A sequence $\epsilon$ is an $n$-tuple

$$
\epsilon=\left(i_{1}, i_{2}, \ldots, i_{n}\right), \quad \text { where } i_{k} \in\{-1,1\} \text { for } 1 \leq k \leq n .
$$

The length $l(\epsilon)$ of a sequence $\epsilon=\left(i_{1}, i_{2}, \ldots, i_{n}\right)$ is given by $l(\epsilon)=n$ and the size $|\epsilon|$ of $\epsilon$ is defined to be the sum $|\epsilon|=i_{1}+\cdots+i_{n}$. If $\epsilon=\left(i_{1}, i_{2}, \ldots, i_{n}\right)$ and $\delta=$ $\left(j_{1}, j_{2}, \ldots, j_{n}\right)$ are two sequences, we write $\epsilon \unrhd \delta$ to mean that

$$
i_{1}+\cdots+i_{k} \geq j_{1}+\cdots+j_{k} \quad \text { for all } k=1, \ldots, n .
$$

For each sequence $\epsilon$, if we denote by 0 the $l(\epsilon)$-tuple consisting entirely of zeros then the sequence $\epsilon$ is admissible if $\epsilon \unrhd 0$.

We denote by $\mathcal{L}_{n}$ the collection of all admissible sequences of length $n$ and by $\mathcal{L}_{n, k} \subset \mathcal{L}_{n}$ the collection of admissible sequences of length $n$ and size $k$ :

$$
\mathcal{L}_{n}=\{\epsilon: l(\epsilon)=n, \epsilon \unrhd 0\} \quad \text { and } \quad \mathcal{L}_{n, k}=\left\{\epsilon \in \mathcal{L}_{n}:|e|=k\right\} .
$$

The relation $\unrhd$ when applied to these sets is called the dominance order.

Definition 2.16 If $\epsilon=\left(i_{1}, i_{2}, \ldots, i_{n}\right)$ is a sequence then we will use the notation $\epsilon \cdot(+1)$ and $\epsilon \cdot(-1)$ to denote the sequence obtained from $\epsilon$ by appending +1 or -1 respectively,

$$
\epsilon \cdot(+1)=\left(i_{1}, i_{2}, \ldots, i_{n},+1\right) \text { and } \epsilon \cdot(-1)=\left(i_{1}, i_{2}, \ldots, i_{n},-1\right) .
$$


Associated to each $\epsilon \in \mathcal{L}_{n}$ is a special element $q_{\epsilon} \in \mathrm{TL}_{n}$. Since these special elements are vertically symmetric, it is easiest to define the top half $t_{\epsilon}$ first.

Definition 2.17 If $\epsilon \in \mathcal{L}_{n}$ and $|\epsilon|=k$ then there is an element $t_{\epsilon} \in \operatorname{TL}(k, n)$ defined inductively by

$$
t_{(1)}=1, \quad t_{\epsilon \cdot(+1)}=\oiint_{\varphi}, \quad \text { and } t_{\epsilon \cdot(-1)}=\overbrace{\uparrow}^{\prime} \text {, }
$$

where the box represents a Jones-Wenzl projector $p_{k}$ and the marshmallow-shaped region represents the element $t_{\epsilon}$. The special element $q_{\epsilon} \in \mathrm{TL}_{n}$ is equal to the top $t_{\epsilon}$ composed with its reverse,

$$
q_{\epsilon}=t_{\epsilon} \bar{t}_{\epsilon} .
$$

The relation $p_{k} p_{k}=p_{k}$ allows us to eliminate one of the two central $p_{k}$ in the definition of $q_{\epsilon}$.

The elements $q_{\epsilon}$ satisfy a recurrence relation.

Lemma 2.18 For each sequence $\epsilon \in \mathcal{L}_{n, k}$, there is a recurrence relation

$$
q_{\epsilon} \sqcup 1=q_{\epsilon \cdot(+1)}+\frac{[k]}{[k+1]} q_{\epsilon \cdot(-1)} .
$$

Proof This follows from applying Equation (2-4) in Section 2.12 to the middle JonesWenzl projector $p_{k}$ of $q_{\epsilon}$ in the definition above.

We will use the special elements $q_{\epsilon} \in \mathrm{TL}_{n}$ to construct idempotents corresponding to the decomposition described in Section 2.10. The following two propositions tell us that there are scalars $f_{\epsilon} \in \mathbb{C}(q)$ such that the collection $p_{\epsilon}=f_{\epsilon} q_{\epsilon}$ satisfies

(1) $p_{\epsilon} p_{\nu}=\delta_{\epsilon, \nu} p_{\epsilon}$

(2) $1_{n}=\sum_{\epsilon \in \mathcal{L}_{n}} p_{\epsilon}$,

where $1_{n} \in \mathrm{TL}_{n}$ is the identity element. The first proposition below tells us that composing $p_{\epsilon}$ and $p_{v}$ when $\epsilon \neq v$ yields zero. Theorem 2.20 will address the second equation and the first equation when $\epsilon=v$.

Proposition 2.19 The special elements $q_{\epsilon} \in \mathrm{TL}_{n}$ defined above are mutually orthogonal,

$$
q_{\epsilon} q_{\nu}=0 \quad \text { for } \epsilon \neq \nu .
$$


Proof Using the definition of $q_{\epsilon}$ found above we can write $q_{\epsilon}=a \bar{a}$ and $q_{\nu}=b \bar{b}$. If $\epsilon \neq v$ then $a=a^{\prime} p_{k}$ and $b=b^{\prime} p_{l}$, where $k \neq l$. The product $q_{\nu} q_{\epsilon}$ contains $p_{k} \bar{a}^{\prime} b^{\prime} p_{l}$ which is equal to zero. By symmetry, $q_{\epsilon} q_{v}$ also vanishes.

In the next proposition, we show that for each $\epsilon \in \mathcal{L}_{n}$, there are constants $f_{\epsilon} \in \mathbb{C}(q)$ and idempotents $p_{\epsilon}=f_{\epsilon} q_{\epsilon}$ which yield the decomposition of identity $1_{n} \in \mathrm{TL}_{n}$ mentioned above.

Theorem 2.20 For each $\epsilon \in \mathcal{L}_{n}$, there are idempotents $p_{\epsilon} \in \mathrm{TL}_{n}$ which satisfy

$$
1_{n}=\sum_{\epsilon \in \mathcal{L}_{n}} p_{\epsilon} .
$$

Moreover, $p_{\epsilon}=f_{\epsilon} q_{\epsilon}$ for some non-zero scalar $f_{\epsilon} \in \mathbb{C}(q)$.

Proof The proof is by induction on the number of strands $n$. When $n$ is 1 the only sequence is $\epsilon=(1)$; we set $f_{\epsilon}=1$ so that $p_{\epsilon}=q_{\epsilon}=1$.

Assume that there is a decomposition of $1_{n-1}$ and place a disjoint strand next to everything. We have

$$
1_{n-1} \sqcup 1=1_{n}=\sum_{\epsilon \in \mathcal{L}_{n-1}} f_{\epsilon} q_{\epsilon} \sqcup 1=\sum_{k} \sum_{\epsilon \in \mathcal{L}_{n-1, k}} f_{\epsilon} q_{\epsilon} \sqcup 1,
$$

in which the elements $p_{\epsilon}=f_{\epsilon} q_{\epsilon}$ are idempotent. The recurrence relation (2-6) implies that

$$
1_{n}=\sum_{k} \sum_{\epsilon \in \mathcal{L}_{n-1, k}} f_{\epsilon}\left(q_{\epsilon \cdot(+1)}+\frac{[k]}{[k+1]} q_{\epsilon \cdot(-1)}\right) .
$$

Setting $p_{\epsilon \cdot(+1)}=f_{\epsilon} q_{\epsilon \cdot(+1)}$ and $p_{\epsilon \cdot(-1)}=f_{\epsilon} \frac{[k]}{[k+1]} q_{\epsilon \cdot(-1)}$ yields the equation in the statement of this proposition.

To show that the $p_{\nu}$ are idempotent, multiply $1_{n}=\sum_{\epsilon} p_{\epsilon}$ on the left with $p_{\nu}$. The previous proposition implies that

$$
p_{\nu}=\sum_{\epsilon} p_{\nu} p_{\epsilon}=p_{\nu} p_{\nu}
$$

Remark 2.21 By Proposition 2.19, $p_{\epsilon} p_{\nu}=0$ when $\epsilon \neq v$ because the projectors $p_{\epsilon}$ differ from the elements $q_{\epsilon}$ by scalars. The construction in the proof of Theorem 2.20 above implies the equation:

$$
p_{\epsilon} \sqcup 1=p_{\epsilon \cdot(+1)}+p_{\epsilon \cdot(-1)} .
$$

By convention $p_{\epsilon}=0$ when $\epsilon \notin \mathcal{L}_{n}$. This equation corresponds to the Clebsch-Gordan rule (2-3) in Section 2.10. 
Remark 2.22 Each idempotent $p_{\epsilon}$ corresponds to projection onto one term $V_{k} \subset$ $m_{k} V_{k} \subset V^{\otimes n}$. The higher-order projectors $p_{n, k}$ correspond to the entire subspace $m_{k} V_{k} \subset V^{\otimes n}$.

Definition 2.23 The $k^{\text {th }}$ higher-order Jones-Wenzl projector $p_{n, k} \in \mathrm{TL}_{n}$ is given by the sum

$$
p_{n, k}=\sum_{\epsilon \in \mathcal{L}_{n, k}} p_{\epsilon}
$$

Remark 2.24 From Proposition 2.19 and Theorem 2.20 it follows that the elements $p_{n, k} \in \mathrm{TL}_{n}$ form a system of mutually orthogonal idempotents. This means that

$$
p_{n, k} p_{n, l}=\delta_{k, l} p_{n, k} \quad \text { and } \quad 1_{n}=\sum_{k} p_{n, k} .
$$

Remark 2.25 Since $p_{n, k}$ is a sum of elements $p_{\epsilon}$ with $|\epsilon|=k$, and each $p_{\epsilon}$ necessarily factors through a Jones-Wenzl projector $p_{k}$, the projector $p_{n, k} \in \mathrm{TL}_{n}$ is a linear combination of diagrams which factor as $b p_{k} a$, where $a \otimes b \in \operatorname{TL}(n, k) \otimes \operatorname{TL}(k, n)$.

Although a definition of the higher-order projectors $p_{n, k}$ was given in Section 2.10, it is often useful to characterize elements intrinsically in terms of their interaction with other elements and gluing operations. This is the definition given below and the one which will lift to the categorical setting in Section 8.

Theorem 2.26 The higher-order Jones-Wenzl projectors $p_{n, k} \in \mathrm{TL}_{n}$ of Definition 2.23 are characterized uniquely by the following properties:

(1) The through-degree $\tau\left(p_{n, k}\right)$ of $p_{n, k}$ is equal to $k$.

(2) The projector $p_{n, k}$ vanishes when the number of turnbacks is sufficiently high: for each $l \in \mathbb{Z}_{+}$and $a \in \operatorname{TL}(n, l)$, if $\tau(a)<k$ then

$$
a p_{n, k}=0 \quad \text { and } \quad p_{n, k} \bar{a}=0 .
$$

(3) The projector $p_{n, k}$ fixes elements of through-degree $k$ up to lower throughdegree terms: for each $l \in \mathbb{Z}_{+}$and $a \in \operatorname{TL}(n, l)$, if $\tau(a)=k$ then

$$
a p_{n, k}=a+b,
$$

where $\tau(b)<k$.

In essence, these properties state that the projectors $p_{n, k}$ control and respect the filtration of TL by through-degree $\tau$, see also the discussion following Definition 2.6. 
Proof We begin by proving that the elements $p_{n, k}$ defined above satisfy properties (1)-(3). Using Remark 2.25 above, we can write $p_{n, k}$ as a sum of the form

$$
p_{n, k}=\sum_{x, y} x p_{k} \bar{y} .
$$

The first property follows from $\tau\left(p_{k}\right)=k$. Now pick some $l \in \mathbb{Z}_{+}$and $a \in \operatorname{TL}(n, l)$.

For the second property, if we assume that $\tau(a)<k$ then

$$
a p_{n, k}=\sum_{x, y} a x p_{k} \bar{y}=0,
$$

since $\tau(a d) \leq \tau(a)<k$ and $p_{k}$ kills diagrams of through-degree less than $k$. For the same reason, $p_{n, k} \bar{a}=0$.

For the third property, if we assume that $\tau(a)=k$ then

$$
a=a 1_{n}=\sum_{l} a p_{n, l}=\sum_{l \leq k} a p_{n, l},
$$

so that rearranging terms gives $a p_{n, k}=a-\sum_{l<k} a p_{n, l}$.

Suppose that $e \in \mathrm{TL}_{n}$ satisfies properties (1)-(3) above. We will show that $e=p_{n, k}$. If $l<k$ then property (2) for $e$ implies that $e p_{n, l}=0$. If $l>k$ then property (2) for $p_{n, l}$ implies that $e p_{n, l}=0$. Therefore,

$$
e=e 1_{n}=\sum_{l} e p_{n, l}=e p_{n, k} .
$$

Property (3) implies that $e p_{n, k}=p_{n, k}+b$, where $\tau(b)<k$. By Theorem 2.20,

$$
e=e p_{n, k}=e p_{n, k}^{2}=\left(p_{n, k}+b\right) p_{n, k}=p_{n, k}^{2}=p_{n, k} .
$$

We continue our discussion of the higher-order projectors with a series of observations.

Proposition 2.27 For each element $a \in \mathrm{TL}(n, m)$, the equation $a p_{n, k}=p_{m, k} a$ holds:

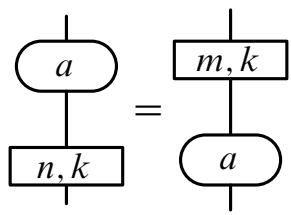

Proof Recall that $1_{m}=\sum_{l} p_{m, l}$ and $1_{n}=\sum_{l} p_{n, l}$. Simplifying the resulting expressions for $1_{m} a p_{n, k}$ and $p_{m, k} a 1_{n}$ gives $a p_{n, k}=p_{m, k} a p_{n, k}=p_{m, k} a$. 
Corollary 2.28 The higher-order projectors $p_{n, k}$ are contained in the center $Z\left(\mathrm{TL}_{n}\right)$ of the Temperley-Lieb algebra.

Corollary 2.29 If $D \in \mathrm{TL}_{n}$ and the through-degree $\tau(D)=l$ so that $D=b a$ where $a \otimes b \in \mathrm{TL}(n, l) \otimes \mathrm{TL}(l, n)$, then

$$
p_{n, k} D=p_{n, k} b a=b p_{l, k} a .
$$

Remark 2.30 This means we can slide a $p_{n, k}$ past some turnbacks onto a fewer number of strands as long as we change it to a $p_{l, k}$. In pictures:

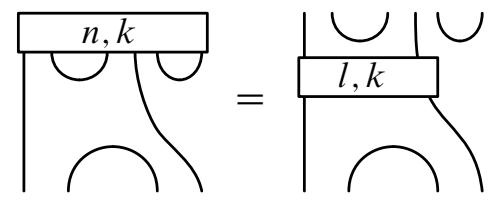

We conclude this section with another definition of $p_{n, k}$. This definition has the value of expressing $p_{n, k}$ in terms of simpler projectors.

Proposition 2.31 The higher Jones-Wenzl projector $p_{n, k}$ satisfies the following recurrence relation:

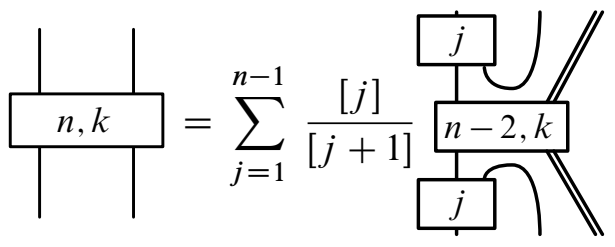

Proof Equation (2-4) implies that

$$
p_{n}=1_{n}-\sum_{j=1}^{n-1} \frac{[j]}{[j+1]}\left(p_{j} \sqcup 1_{n-j}\right) e_{j}\left(p_{j} \sqcup 1_{n-j}\right) .
$$

Applying $p_{n, k}$ to both sides of this equation gives

$$
p_{n, k}=\sum_{j=1}^{n-1} \frac{[j]}{[j+1]} p_{n, k}\left(p_{j} \sqcup 1_{n-j}\right) e_{j}\left(p_{j} \sqcup 1_{n-j}\right),
$$

which becomes the desired equation after applying Proposition 2.27.

Iterating this formula expresses $p_{n, k}$ purely in terms of Jones-Wenzl projectors $p_{l}$. 


\section{Categorification of the Temperley-Lieb category}

In this section we recall Dror Bar-Natan's graphical formulation [2] of the Khovanov categorification [16; 17]. We follow the same conventions as [8].

There is a pre-additive category $\operatorname{Pre}-\operatorname{Cob}(n)$ whose objects are isotopy classes of formally $q$-graded Temperley-Lieb diagrams with $2 n$ boundary points. The morphisms are given by $\mathbb{Z}[\alpha]$-linear combinations of isotopy classes of orientable cobordisms, decorated with dots, and bounded in $D^{2} \times[0,1]$ between two disks containing such diagrams. The degree of a cobordism $C: q^{i} A \rightarrow q^{j} B$ is given by

$$
\operatorname{deg}(C)=\operatorname{deg}_{\chi}(C)+\operatorname{deg}_{q}(C),
$$

where the topological degree $\operatorname{deg}_{\chi}(C)=\chi(C)-n$ is given by the Euler characteristic of $C$ and the $q$-degree $\operatorname{deg}_{q}(C)=j-i$ is given by the relative difference in $q$-gradings. The maps $C$ used throughout the paper will satisfy $\operatorname{deg}(C)=0$. The formal $q$-grading will be chosen to cancel the topological grading.

When working with chain complexes, every object will also contain a homological grading and every map will have an associated homological degree. This homological degree, or $t$-degree, is not part of the definition $\operatorname{deg}(C)$. We may refer to degree as internal degree in order to differentiate between degree and homological degree.

We impose the relations below to obtain a new category $\operatorname{Cob}(n)$ as a quotient of the category $\operatorname{Mat}(\operatorname{Pre}-\operatorname{Cob}(n))$ formed by allowing direct sums of objects and maps between them:
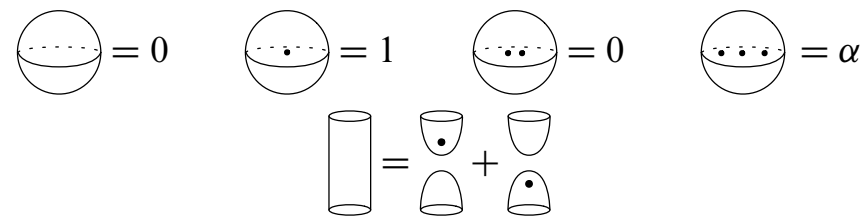

The dot is determined by the relation that two times a dot is equal to a handle. When $\alpha=0$ the cylinder or neck cutting relation implies that closed surfaces $\Sigma_{g}$ of genus $g>3$ evaluate to 0 . In what follows we will let $\alpha$ be a free variable and absorb it into our base ring $\left(\Sigma_{3}=8 \alpha\right)$. One can think of $\alpha$ as a deformation parameter, see [2].

The categories $\operatorname{Cob}(n)$ fit together in much the same way as the Temperley-Lieb algebras $\mathrm{TL}_{n}$. There is an inclusion

$$
-\sqcup 1_{m-n}: \operatorname{Cob}(n) \rightarrow \operatorname{Cob}(m) \quad \text { when } \quad n<m,
$$

which is obtained by placing $m-n$ disjoint vertical line segments to the right of each object. On morphisms, $f \sqcup 1_{m-n}$ is defined to be the union of $f$ and $m-n$ copies of an identity cobordism. If $m=n$ then the empty set is used. 
There is a category $\operatorname{Cob}(m, n)$ with objects corresponding to diagrams in $\operatorname{TL}(m, n)$, so that $\operatorname{Cob}(n)=\operatorname{Cob}(n, n)$. There is a composition

$$
\otimes: \operatorname{Cob}(n, k) \times \operatorname{Cob}(k, l) \rightarrow \operatorname{Cob}(n, l), \quad A \times B \mapsto B \otimes A,
$$

obtained by gluing all diagrams and morphisms along the $k$ boundary points and $k$ boundary intervals respectively. Pictorially:

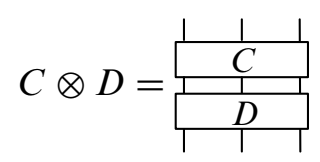

This composition makes the collection of categories $\operatorname{Cob}(n, k)$ into a 2-category Cob. The relationship between Cob and the Temperley-Lieb category TL can be described using the Grothendieck group functor $\mathrm{K}_{0}$.

Theorem 3.1 The 2-category Cob categorifies the Temperley-Lieb category TL. There are isomorphisms

$$
\mathrm{TL}(n, k) \cong \mathrm{K}_{0}(\operatorname{Cob}(n, k)) \otimes_{\mathbb{Z}\left[q, q^{-1}\right]} \mathbb{C}(q)
$$

which commute with composition.

These isomorphisms commute with the compositions of Equation (3-1). For more detail, see $[2 ; 17 ; 9]$.

Definition 3.2 The category of chain complexes of cobordisms will be denoted by $\operatorname{Kom}(n, m)$,

$$
\operatorname{Kom}(n, m)=\operatorname{Kom}(\operatorname{Cob}(n, m)) .
$$

Unless otherwise stated chain complexes are bounded from below in homological degree. All chain complexes produced in what follows will have differentials with components having internal degree zero. Restricting to the subcategory of chain complexes with degree zero differentials yields a well-behaved Grothendieck group $\mathrm{K}_{0}(\operatorname{Kom}(n, k))$; see [8].

The category of chain complexes can be enriched to form a differential graded category.

Definition 3.3 There is a differential graded category, $\operatorname{Kom}^{*}(n, m)$, which has the same objects as the category $\operatorname{Kom}(n, m)$ but with morphisms that are given by allowing maps of all homological degrees.

If $f \in \operatorname{Hom}^{m}(A, B)$ is a map of homological degree $m$ then $d(f)=[d, f] \in$ $\operatorname{Hom}^{m+1}(A, B)$. Each collection of morphisms from $A$ to $B$ in $\operatorname{Kom}^{*}(n, m)$, denoted by $\operatorname{Hom}^{*}(A, B)$, is a chain complex and the differential $d$ is a derivation with respect to composition of maps. 


\subsection{Grading shifts}

In this section we remind the reader how degree shifts are denoted. Each chain complex can be shifted in $q$-degree or $t$-degree.

If $A$ is a chain complex then $t A$ will denote the chain complex shifted in homological degree by 1 ,

$$
(t A)_{i}=A_{i-1} \quad \text { and } \quad d_{t A}=-d_{A} .
$$

We will use $q A$ to denote the chain complex satisfying $\operatorname{deg}_{q}(q B)=\operatorname{deg}_{q}(B)+1$, where $B \in \operatorname{Pre}-\operatorname{Cob}(n)$ corresponds to a summand of $A$. See Section 3 for a discussion of $q$-degree.

If $C \in \operatorname{Kom}(n, m)$ is a chain complex and $f(q) \in \mathbb{Z}\left[q^{-1}\right] \llbracket q \rrbracket$ is a power series then we will write $[f(q) C]$ for an iterated cone of chain complexes $A_{0}, A_{1}, \ldots$ in which $A_{i}=C$ for all $i$. The relation

$$
[f(q) C]=f(q) \cdot[C]
$$

holds in the Grothendieck group $\mathrm{K}_{0}(\operatorname{Kom}(n, m))$.

Definition 3.5 If $C$ is a chain complex of the form $Q_{\epsilon}$, where $\mathrm{K}_{0}\left(Q_{\epsilon}\right)=q_{\epsilon}$, then we will consistently omit a product of terms of the form $[k] /[k+1]$ from the bracket notation. Usually,

$$
\left[Q_{\epsilon}\right]=\left[f_{\epsilon} Q_{\epsilon}\right],
$$

where $f_{\epsilon}$ is defined in the proof of Theorem 2.20.

\subsection{Universal projectors}

The most important object in the categories defined above is an idempotent chain complex $P_{n} \in \operatorname{Kom}(n)$ which categorifies the Jones-Wenzl projector $p_{n} \in \mathrm{TL}_{n}$. The chain complexes $P_{n}$ will be used repeatedly in later sections in order to construct the projectors $P_{\epsilon}$ and $P_{n, k}$ that correspond to the elements $p_{\epsilon}$ and $p_{n, k}$ introduced in Section 2.

Theorem 3.7 (Cooper and Krushkal [8]) There exists a chain complex $P_{n} \in \operatorname{Kom}(n)$, called the universal projector, such that:

(1) $P_{n}$ is positively graded with differential having internal degree zero.

(2) The identity diagram appears only in homological degree zero and only once.

(3) For each diagram $D$ which is not the identity, the chain complex $P_{n} \otimes D$ is contractible. 
These three properties characterize $P_{n}$ uniquely up to homotopy.

See also [11;24], and [5; 28] for related ideas.

We conclude this section with a lemma. This lemma plays an important role in the proof of Theorem 7.1.

Lemma 3.8 If $P_{n} \in \operatorname{Kom}(n)$ is a projector then there is a twisted complex

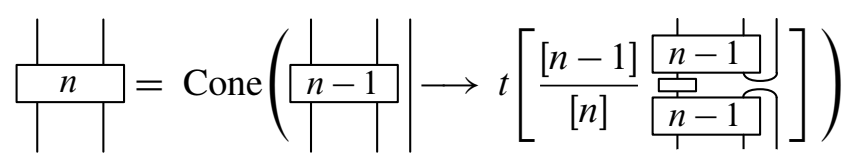

which is also a projector.

Proof The proof follows from tensoring the Frenkel-Khovanov complex of [8] for $P_{n}$ with $\left(P_{n-2} \sqcup 1_{2}\right) \otimes e_{n-1} \otimes\left(P_{n-1} \sqcup 1_{1}\right)$ then contracting portions of the subcomplex consisting of projectors containing turnbacks.

\section{Twisted complexes and operations on twisted complexes}

\subsection{Twisted complexes}

In this section we recall the definition of the category $\operatorname{Tw} \mathcal{A}$ of twisted complexes over a differential graded category $\mathcal{A}$, see $[4 ; 21]$. The reader may assume that $\mathcal{A}=\operatorname{Kom}^{*}(n, m)$, see Definition 3.3.

Our main construction in Section 7 will occur in the category of twisted complexes. Informally, the definitions in this section codify situations in which the objects of study are chain complexes $M$ with a decreasing filtration

$$
M=F^{0} M \supseteq F^{1} M \supseteq F^{2} M \supseteq \cdots
$$

and a splitting $F^{i+1}(M)=G_{i+1} \oplus F^{i}(M)$ as graded objects. Maps are required to respect this filtration.

The definitions presented here are variations on standard ones which allow one to work with categories of twisted complexes that are unbounded and indexed by countable sets (such as $\mathbb{Z}_{+}$). This is accomplished by requiring that maps are lower triangular, see Definition 4.6 below. 
Definition 4.2 A twisted complex over $\mathcal{A}$ is a collection

$$
\left\{\left(E_{i}\right), q_{i j}: E_{i} \rightarrow E_{j}\right\}, \quad \text { where } i \in \mathbb{Z}_{+},
$$

consisting of objects $E_{i} \in \mathcal{A}$ and maps $q_{i j}$ of degree 1 which satisfy $q_{i j}=0$ for $i \geq j$, and the equation

$$
(-1)^{j} d_{\mathcal{A}}\left(q_{i j}\right)+\sum_{k} q_{k j} \circ q_{i k}=0
$$

Definition 4.3 Twisted complexes which satisfy the condition that $q_{i j}=0$ when $i \geq j$ are called one-sided.

Remark 4.4 All infinite twisted complexes will be one-sided. The resolution of identity $R_{n}$ in Theorem 7.4 is a one-sided twisted complex.

It will be convenient later to use ordered sets besides $\mathbb{Z}_{+}$to index components of twisted complexes. In particular, the set of sequences $\mathcal{L}_{n}$ together with the dominance order described in Definition 2.15 will be used throughout Section 7. In general, it will be clear from context when this is done.

Definition 4.5 $(\operatorname{Tw} \mathcal{A})$ The one-sided twisted complexes form a differential graded category. If $A=\left\{\left(A_{i}\right), a_{i j}\right\}$ and $B=\left\{\left(B_{i}\right), b_{i j}\right\}$, then degree $k$ maps are those that intertwine the diagrams formed by $A$ and $B$,

$$
\operatorname{Hom}_{\mathrm{Tw} \mathcal{A}}^{k}(A, B)=\prod_{i \leq j} \operatorname{Hom}_{\mathrm{Kom} *(n, m)}^{k+i-j}\left(A_{i}, B_{j}\right) .
$$

In other words, morphisms $f: A \rightarrow B$ are collections $\left\{f_{i j}\right\}$ of maps having the appropriate degree which satisfy $f_{i j}=0$ unless $i \leq j$. The composition of morphisms is defined in terms of components by the equation

$$
(f \circ g)_{i j}=\sum_{i \leq k \leq j} f_{k j} \circ g_{i k}
$$

If $f \in \operatorname{Hom}^{*}(A, B)$ is given by $\left\{f_{i j}\right\}$, then the equation

$$
(d f)_{i j}=(-1)^{j} d_{\mathcal{A}}\left(f_{i j}\right)+\sum_{k} b_{k j} \circ f_{i k}-(-1)^{|f|} f_{k j} \circ q_{i k}
$$

determines a differential which makes $\operatorname{Tw} \mathcal{A}$ into a differential graded category. 
The categories Tw $\operatorname{Kom}^{*}(n, m)$ are examples of pre-triangulated categories. Pretriangulated categories can be seen as an alternative to triangulated categories because every such category $\mathcal{A}$ yields a triangulated category $H^{0}(\mathcal{A})$, see [4].

If $\left\{E_{i}\right\} \subset \operatorname{Kom}(\mathcal{C})$ is a collection of non-negatively graded chain complexes, then as graded objects, $\prod_{i \geq 0} t^{i} E_{i} \cong \bigoplus_{i \geq 0} t^{i} E_{i}$ since the direct product is finite in each degree. This allows us to flatten each twisted complex $A=\left\{\left(A_{i}\right), a_{i j}\right\}$ to a chain complex $\operatorname{Tot}(A)$ by summing together the individual components $A_{i}$ of $A$.

Definition 4.6 If $\mathcal{A}=\operatorname{Kom}(\mathcal{C})$ is the category of non-negatively graded chain complexes over an additive category $\mathcal{C}$, then there is a $\mathrm{dg}$ functor

$$
\text { Tot: } \operatorname{Tw} \mathcal{A} \rightarrow \mathcal{A}
$$

from twisted complexes to complexes, defined on objects $\left\{\left(E_{i}\right), q_{i j}\right\} \in \mathrm{Tw} \mathcal{A}$ by

$$
\operatorname{Tot}\left(\left\{\left(E_{i}\right), q_{i j}\right\}\right)=\left\{\bigoplus_{i \geq 0} t^{i} E_{i}, d\right\}, \quad \text { where } d=\left(\begin{array}{cccc}
d_{E_{0}} & & & \\
q_{01} & -d_{E_{1}} & & \\
q_{02} & q_{12} & d_{E_{2}} & \\
\vdots & \vdots & \vdots & \ddots
\end{array}\right)
$$

and on morphisms $f=\left\{f_{i j}\right\}$ by

$$
\operatorname{Tot}(f)=\left(\begin{array}{cccc}
f_{00} & & & \\
f_{01} & f_{11} & & \\
f_{02} & f_{12} & f_{22} & \\
\vdots & \vdots & \vdots & \ddots
\end{array}\right)
$$

Remark 4.7 The condition that $d_{\mathrm{Tot}(A)}^{2}=0$ is implied by Equation (4-1) above.

Remark 4.8 The functor Tot defined above preserves homotopy equivalences. In particular, for all $X, Y \in \operatorname{Tw} \mathcal{A}, X \simeq Y \Rightarrow \operatorname{Tot}(X) \simeq \operatorname{Tot}(Y)$.

Remark 4.9 If $\mathcal{C}$ is an additive category which contains countable direct products, and $\mathcal{A}$ is the differential graded category of possibly unbounded chain complexes over $\mathcal{C}$, then we can define a $\mathrm{dg}$ functor

$$
\operatorname{Tot}^{\Pi}: \operatorname{Tw} \mathcal{A} \rightarrow \mathcal{A}
$$

using the formulas above with $\oplus$ replaced by $\Pi$.

Definition 4.10 (Convolution) If a chain complex $A$ is the total complex of some twisted complex $\left\{\left(E_{i}\right), q_{i j}\right\}$, then we say $A$ is a convolution of $\left\{\left(E_{i}\right), q_{i j}\right\}$. 
Example 4.11 In Lemma 4.26 the twisted complex $T$ pictured below is considered:

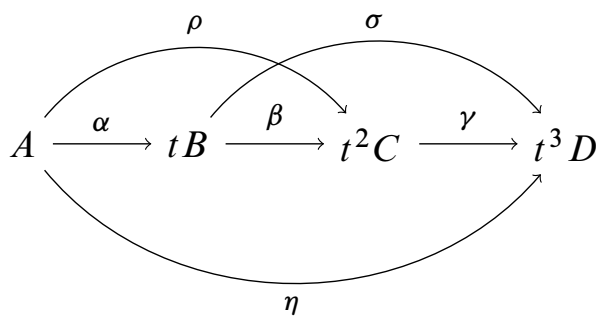

Each object $A, B, C$ and $D$ is a chain complex. The convolution $\operatorname{Tot}(T)$ is the chain complex $A \oplus t B \oplus t^{2} C \oplus t^{3} D$ with differential

$$
d_{\operatorname{Tot}(T)}=\left(\begin{array}{ccccc}
d_{A} & & & \\
\alpha & -d_{B} & & \\
\rho & \beta & d_{C} & \\
\eta & \sigma & \gamma & -d_{D}
\end{array}\right) .
$$

This twisted complex is one-sided with respect to the order of the letters appearing in the alphabet.

The notion of hull defined below formalizes the idea of the subcategory of all chain complexes built out of iterated extensions of elements of some fixed set of chain complexes.

Definition 4.12 If $\mathcal{E}=\left\{A_{1}, \ldots, A_{r}\right\} \subset \operatorname{Kom}(\mathcal{C})$ is a collection of chain complexes, then the hull $\langle\mathcal{E}\rangle \subset \operatorname{Kom}(\mathcal{C})$ is the smallest strictly full additive subcategory containing each $A_{i}$ and closed under convolution.

In particular, if $\left\{\left(E_{i}\right), q_{i j}\right\} \in \operatorname{Tw} \operatorname{Kom}(\mathcal{C})$ satisfies $E_{i} \in\langle\mathcal{E}\rangle$ for all $i \in \mathbb{Z}_{+}$, then $\operatorname{Tot}\left(\left\{\left(E_{i}\right), q_{i j}\right\}\right) \in\langle\mathcal{E}\rangle$.

Definition 4.13 Suppose that $A=\left\{\left(A_{i}\right), a_{i j}\right\}$ and $B=\left\{\left(B_{i}\right), b_{i j}\right\}$ are twisted complexes and $f=\left\{f_{i j}\right\}: A \rightarrow B$ is a degree-zero cycle then the cone of $f$ is the twisted complex given by

$$
\operatorname{Cone}(f)=\left\{\left(A_{i} \oplus B_{i-1}\right),\left(\begin{array}{cc}
a_{i j} & 0 \\
f_{i, j-1} & -b_{i-1, j-1}
\end{array}\right)\right\} .
$$

Remark 4.14 The condition that Cone $(f)$ is a twisted complex is equivalent to the requirement that $f$ is a degree zero cycle in the definition above. 
Remark 4.15 If $A, B$ are twisted complexes over a category of chain complexes and $f: A \rightarrow B$ a degree zero cycle, then

$$
\operatorname{Tot}(\operatorname{Cone}(f))=\operatorname{Cone}(\operatorname{Tot}(f)) \text {. }
$$

Definition 4.16 Suppose that $Y=\left\{\left(Y_{i}\right), y_{i j}\right\}$ is a twisted complex indexed by $\mathbb{Z}_{+}$. For each $r, s \in \mathbb{Z}_{+}$with $r \leq s$, the $[r, s]$-truncation of $Y$ is given by

$$
Y_{[r, s]}=\left\{\left(T_{i}\right), t_{i j}\right\},
$$

where $T_{i}=Y_{i}, t_{i j}=y_{i j}$ when $i, j \in[a, b]$, and $T_{i}=0, t_{i j}=0$ when $i, j \notin[r, s]$.

The lemma below says that a twisted complex is determined by its truncations, and each truncation is an iterated mapping cone. For simplicity of notation, we restrict to $\mathbb{Z}_{+}$-indexed twisted complexes over the categories $\operatorname{Kom}^{*}(n)$.

Lemma 4.17 If $\left\{\left(E_{i}\right), q_{i j}\right\}$ is a twisted complex over $\operatorname{Kom}^{*}(n)$, then for each integer $s \geq 0$,

$$
\operatorname{Tot}\left(Y_{[0, s]}\right)=\operatorname{Cone}\left(\operatorname{Tot}\left(Y_{[0, s-1]}\right) \stackrel{\delta}{\longrightarrow} t^{s-1} E_{s}\right),
$$

where $\delta=\left(\begin{array}{llll}q_{0, s} & q_{1, s} \ldots & \ldots & q_{s-1, s}\end{array}\right)$ is a chain map of degree zero. Conversely, if we have chain complexes $C_{s}$ and maps $\delta_{s}: C_{s} \rightarrow t^{s} E_{s+1}$ such that $C_{s+1}=\operatorname{Cone}\left(\delta_{s}\right)$, then there is a unique twisted complex $Y$ such that $C_{s}=\operatorname{Tot}\left(Y_{[0, s]}\right)$.

Recall that an object $E$ in a $\operatorname{dg}$ category $\mathcal{A}$ is contractible when the map $\operatorname{Id}_{E}$ is a boundary in the mapping space $\operatorname{End}_{\mathcal{A}}(E)$. The next lemma is a useful tool for showing that certain filtered chain complexes are contractible.

Lemma 4.18 If $\left\{E_{i}\right\} \subset \mathcal{A}$ is a collection of contractible objects then each twisted complex $\left\{\left(E_{i}\right), q_{i j}\right\}$ is contractible.

Remark 4.19 One subtlety to keep in mind is that the corresponding result for chain complexes only holds in situations where convolution Tot is defined, eg over a category of non-negatively graded chain complexes or a category of (possibly unbounded) chain complexes over an additive category containing countable direct products.

The following theorem says that the $\mathrm{dg}$ subcategory of $\mathcal{A}$ determined by the hull of $\mathcal{E}$ is controlled by the dg algebra of Hom-spaces between objects in $\mathcal{E}$.

Theorem 4.20 [4] If $\mathcal{E}$ is a collection of objects in a pre-triangulated category $\mathcal{A}$, then the category of differential graded modules over the algebra

$$
E=\bigoplus_{i, j} \operatorname{Hom}^{*}\left(E_{i}, E_{j}\right)
$$

is equivalent to the category of $\langle\mathcal{E}\rangle$. 


\subsection{Operations on twisted complexes}

In this section we introduce a number of lemmas and notations which will be used repeatedly throughout Sections 6 and 7.

We will use the following proposition in Theorem 7.1 to construct the chain complexes $P_{\epsilon}$.

Proposition 4.22 (Obstruction theory) For each pair of chain maps $\alpha: A \rightarrow B$ and $\beta: B \rightarrow C$, there is a chain map $\gamma: \operatorname{Cone}(\alpha) \rightarrow t C$ of the form $\gamma=(-h \beta)$, where $\beta \circ \alpha=d_{C} \circ h$ if and only if $\beta \circ \alpha \simeq 0$. Moreover, if $\operatorname{Hom}^{*}(A, C) \simeq 0$ then $\beta \circ \alpha \simeq 0$ and the map $\gamma$ is unique up to homotopy.

Proof The associated homotopy category $\mathrm{Ho}(\mathrm{Kom})$ is triangulated. There is an exact triangle $A \rightarrow B \rightarrow \operatorname{Cone}(\alpha)$. Applying the functor $\operatorname{Hom}(-, C)$ yields a long exact sequence

$$
\cdots \rightarrow \operatorname{Hom}^{i}(\operatorname{Cone}(\alpha), C) \rightarrow \operatorname{Hom}^{i}(B, C) \rightarrow \operatorname{Hom}^{i}(A, C) \rightarrow \cdots .
$$

If $\beta \circ \alpha \simeq 0$ then $\alpha^{*}(\beta)=0$ and exactness implies the existence of $\gamma$. One can check that $\gamma$ is given by the map above between chain complexes. Uniqueness of $\gamma$ is implied by exactness on the other side.

Remark 4.23 The uniqueness of the lifts $\gamma$ in the proposition above will guarantee that there is exactly one choice at each stage in the construction of the projectors $P_{\epsilon}$, see Theorem 7.1 and Corollary 7.2.

It will be useful to add a contractible chain complex to a chain complex using the Cone construction.

Lemma 4.24 (Substitution) Let $A, B, C, D$ be chain complexes and $f: B \rightarrow D$ a chain map. Then we have
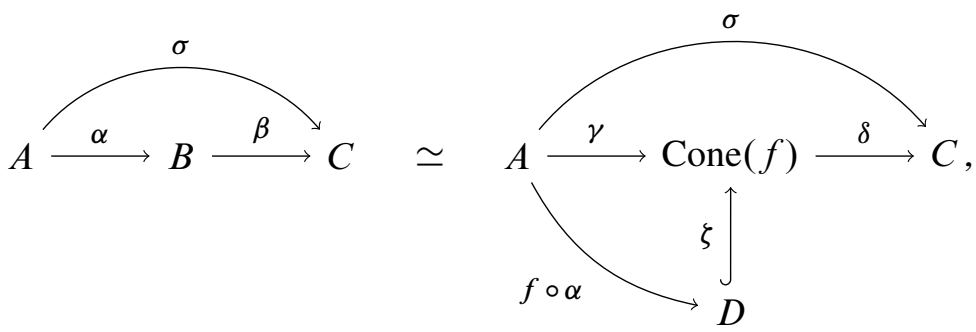

where $\gamma=\left(\begin{array}{l}\alpha \\ 0\end{array}\right), \delta=\left(\begin{array}{ll}\beta & 0\end{array}\right)$ and $\zeta=\left(\begin{array}{c}0 \\ \text { Idd }\end{array}\right)$. 
The following corollary is an inductive version of the previous lemma.

Corollary 4.25 (Inductive substitution) Suppose that $A=\left\{\left(A_{i}\right), q_{i j}\right\}$ is a one-sided twisted complex and there are maps $f_{i}: A_{i} \rightarrow C_{i}$. Then $A \simeq\left\{\left(C_{i} \hookrightarrow B_{i}\right), p_{i j}\right\}$, where $B_{i}=\operatorname{Cone}\left(f_{i}\right)$.

Note that we may have $C_{i}=0$ for some $i$ in the above corollary.

The next lemma allows us to remove arrows between objects in a twisted complex when the Hom-space between these objects is contractible (as in Theorem 5.13).

Lemma 4.26 (Combing) If $\beta \in \operatorname{Hom}^{*}(B, C)$ is a boundary, then
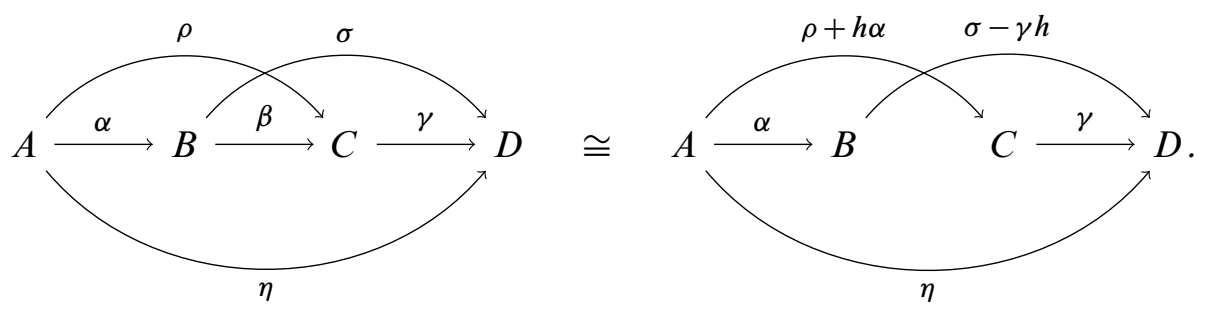

In words, we may remove $\beta$ from the right-hand side. However, in doing so, we perturb the differential by arrows which factor through $\alpha$ or $\gamma$. Note that if $\operatorname{Hom}^{*}(B, C) \simeq 0$, then every cycle $f \in \operatorname{Hom}^{*}(B, C)$ is a boundary.

Proof Since $\beta$ is a boundary there exists a homotopy $h: B \rightarrow C$ such that $\beta=d h-h d$. This allows us to define maps $\varphi=\varphi^{1}, \varphi^{-1}$ where

$$
\varphi^{ \pm 1}=\left(\begin{array}{cccc}
\operatorname{Id}_{A} & & & \\
& \operatorname{Id}_{B} & & \\
& \pm h & \operatorname{Id}_{C} & \\
& & & \operatorname{Id}_{D}
\end{array}\right) .
$$

Notice that $\varphi \varphi^{-1}=$ Id and $\varphi^{-1} \varphi=$ Id. If $d$ is the differential on the left-hand side then $\varphi d_{\operatorname{Tot}(T)} \varphi^{-1}$ is the differential on the right-hand side.

\section{Computing spaces of maps using duality}

In this section we recall a duality for $\operatorname{Hom}-$ spaces inside of the category $\operatorname{Kom}(n, m)$. For each sequence $\epsilon \in \mathcal{L}_{n}$, chain-level analogues $Q_{\epsilon} \in \operatorname{Kom}(n)$ of the elements $q_{\epsilon} \in \mathrm{TL}_{n}$ found in Section 2.14 are introduced. In Theorem 5.13 the duality statement is used to prove that Hom-spaces between convolutions of $Q_{\epsilon}$ and $Q_{\nu}$ respect the dominance order. 


\subsection{Duality}

Notation 5.2 Denote by $\operatorname{Kom}(n)^{b}$ the subcategory of $\operatorname{Kom}(n)$ consisting of chain complexes which are bounded on both sides in homological degree.

Definition $5.3\left(C^{\vee}\right)$ If $C \in \operatorname{Kom}(n)^{b}$ then the dual complex $C^{\vee}$ is obtained by reflecting all of the diagrams in the chain complex about the $x$-axis and reversing both the quantum and homological gradings.

Remark 5.4 When defining the invariants of tangles which live in $\operatorname{Kom}(n)^{b}$ (see [2; 17]) the chain complex associated to a negative crossing can be obtained from the chain complex associated to a positive crossing by applying this functor.

Remark 5.5 One can show that $\left(C^{\vee}\right)^{\vee} \cong C$ and that $-^{\vee}$ : $\operatorname{Kom}(n)^{b} \rightarrow \operatorname{Kom}(n)^{b}$ preserves homotopy.

Our primary interest in $-^{\vee}$ stems from its behavior with respect to the pairing

$$
\operatorname{Kom}(n)^{b} \times \operatorname{Kom}(n)^{b} \rightarrow \operatorname{Kom}(0)^{b}, \quad(A, B) \mapsto \operatorname{Hom}^{*}(A, B) .
$$

The computation of Hom-spaces in $\operatorname{Kom}(n)^{b}$ can be simplified using the planar algebra trick which is illustrated below:

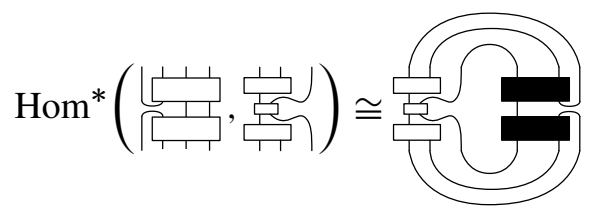

The boxes above represent choices of chain complexes in $\operatorname{Kom}(n)^{b}$ so that on the left-hand side of this equation is the chain complex of maps between the two objects in the differential graded category $\operatorname{Kom}^{*}(n)^{b}$. On the right-hand side of this equation is the chain complex in $\operatorname{Kom}(0)^{b}$ formed by dualizing the first of the two objects and then connecting its free end points to those of the second object. This is identified with the chain complex of abelian groups on the left after applying the functor $\operatorname{Hom}(\varnothing,-)$.

The theorem below contains a precise statement of the general case.

Theorem 5.6 For all $A, B \in \operatorname{Kom}(n)^{b}$, the Markov trace of the chain complex $B \otimes$ $A^{\vee} \in \operatorname{Kom}(n)^{b}$ computes the extended Hom-space from $A$ to $B$,

$$
\operatorname{Hom}^{*}(A, B) \cong \operatorname{Tr}\left(B \otimes A^{\vee}\right) \text {. }
$$


Proof If $f: D \rightarrow D^{\prime}$ is a map between diagrams $D$ and $D^{\prime}$ in $\operatorname{Cob}(n)$ then there is a canonical way to construct an element of $D^{\prime \vee} \otimes D$. This commutes with convolutions and so respects the differential.

Remark 5.7 This duality has been explored in [7, Thm 1.3], in [23] this duality was denoted by $-\diamond$, and in $[5]$ it is denoted by $\mathbb{D}(-)$. Moreover, in each rigid monoidal category there is an isomorphism $\operatorname{Hom}\left(1, A^{\vee} \otimes B\right) \rightarrow \operatorname{Hom}(A, B)$, see [1]. In our setting we can identify the left-hand side with the chain complex determined by the Markov trace.

Since the category $\operatorname{Kom}(n)^{b}$ of bounded complexes is closed under the duality $-^{\vee}$ it has an extra bit of symmetry which is lacking in the category $\operatorname{Kom}(n)$. One way to use the theorem above in our context is to allow one term in the Hom-pairing to be a chain complex in $\operatorname{Kom}(n)$ and require the other term to be a chain complex in $\operatorname{Kom}(n)^{b}$ :

$$
\operatorname{Kom}(n)^{b} \times \operatorname{Kom}(n) \rightarrow \operatorname{Kom}(0) \quad \text { or } \quad \operatorname{Kom}(n) \times \operatorname{Kom}(n)^{b} \rightarrow \operatorname{Kom}(0) .
$$

Then Theorem 5.6 above continues to hold. This is all that is necessary for the proof of Theorem 5.13 below. For an alternative viewpoint, see [14].

\subsection{Categorical quasi-idempotents}

In this section we associate to each sequence $\epsilon \in \mathcal{L}_{n}$ a special chain complex $Q_{\epsilon} \in$ $\operatorname{Kom}(n)$. This construction is directly analogous to the definition of $q_{\epsilon}$ in Section 2.14.

Definition 5.9 If $\epsilon \in \mathcal{L}_{n}$ and $|\epsilon|=k$, then there is an element $T_{\epsilon} \in \operatorname{Kom}(k, n)$ which is defined inductively by

$$
T_{(1)}=1, \quad T_{\epsilon \cdot(+1)}=\oiint_{\varphi}, \text { and } T_{\epsilon \cdot(-1)}=\overbrace{\varphi}^{\prime} \text {, }
$$

where the box represents a universal projector $P_{k}$ (Theorem 3.7) and the marshmallowshaped region represents the element $T_{\epsilon}$. The special element $Q_{\epsilon} \in \operatorname{Kom}(n)$ is equal to the top $T_{\epsilon}$ composed with its reverse,

$$
Q_{\epsilon}=T_{\epsilon} \otimes \bar{T}_{\epsilon} .
$$

In other words, replacing $p_{k}$ with $P_{k}$ in the definition of $q_{\epsilon}$ gives us $Q_{\epsilon}$. The graded Euler characteristic of $Q_{\epsilon}$ is the element of $\mathrm{TL}_{n}$ obtained from $q_{\epsilon}$ after identifying its coefficients with elements of $\mathbb{Z} \llbracket q \rrbracket\left[q^{-1}\right]$.

The chain complexes $Q_{\epsilon}$ will be used extensively in Sections 6 and 7. 


\subsection{Hulls of $Q_{\epsilon}$ are perpendicular}

Before stating the main theorem in this section, we must introduce a lemma which will be used in its proof.

Lemma 5.11 If $N \in \operatorname{Kom}(n)$, then the collection of complexes annihilated by $N$,

$$
\operatorname{Ann}(N)=\{M \in \operatorname{Kom}(n): M \otimes N \simeq 0\},
$$

is closed under convolution.

Proof If $\left\{\left(E_{i}\right), q_{i j}\right\}$ is a twisted complex with $E_{i} \in \operatorname{Ann}(N)$ then by Lemma 4.18,

$$
\operatorname{Tot}\left(\left\{\left(E_{i}\right), q_{i j}\right\}\right) \otimes N \cong \operatorname{Tot}\left(\left\{\left(E_{i} \otimes N\right), q_{i j} \otimes \operatorname{Id}_{N}\right\}\right) \simeq 0 .
$$

Remark 5.12 In particular, if $Q_{\epsilon} \otimes N \simeq 0$ then $\left\langle Q_{\epsilon}\right\rangle \subset \operatorname{Ann}(N)$ and so each element $\left[Q_{\epsilon}\right] \in\left\langle Q_{\epsilon}\right\rangle$ in the hull of $Q_{\epsilon}$ satisfies $\left[Q_{\epsilon}\right] \otimes N \simeq 0$.

The following theorem tells us that each convolution of $Q_{\epsilon}$ is perpendicular to each convolution of $Q_{\nu}$ with respect to the Hom-pairing when $\epsilon \not \Perp v$. This is used in Theorem 7.1, in conjunction with Proposition 4.22, to inductively construct the projectors $P_{\epsilon}$.

Theorem 5.13 If $\epsilon, v \in \mathcal{L}_{n}$ are sequences and $\epsilon \not \Perp v$, then

$$
\operatorname{Hom}^{*}\left(\left[Q_{\epsilon}\right],\left[Q_{\nu}\right]\right) \simeq 0
$$

for each $\left[Q_{\epsilon}\right] \in\left\langle Q_{\epsilon}\right\rangle$ and $\left[Q_{\nu}\right] \in\left\langle Q_{\nu}\right\rangle$.

Proof Suppose that $n \in \mathbb{Z}_{+}$and $N=\left[Q_{\nu}\right]^{n}$ is the $n^{\text {th }}$ chain group of the chain complex $\left[Q_{v}\right]$. The condition $\epsilon \not \Perp v$ implies that there is an $i$ such that

$$
\epsilon_{1}+\cdots+\epsilon_{i}>v_{1}+\cdots+v_{i} .
$$

Let $k=\epsilon_{1}+\cdots+\epsilon_{i}$ and $l=v_{1}+\cdots+v_{i}$. By definition of $Q_{v}$, every summand $a$ of $N=\left[Q_{\nu}\right]^{n}$ can be written as

$$
a=c \otimes\left(b \sqcup 1_{n-i}\right) \quad \text { for some } \quad b \in \mathrm{TL}(i, l),
$$

and $b$ satisfies $Q_{\epsilon} \otimes\left(b \sqcup 1_{n-i}\right)^{\vee} \simeq 0$ (for the same reasons as Proposition 2.19). Hence, $Q_{\epsilon} \otimes N^{\vee} \simeq 0$. Lemma 5.11 and Theorem 5.6 imply that

$$
\operatorname{Hom}^{*}\left(\left[Q_{\epsilon}\right],\left[Q_{\nu}\right]^{n}\right)=\operatorname{Hom}^{*}\left(\left[Q_{\epsilon}\right], N\right)=\operatorname{Hom}^{*}\left(\left[Q_{\epsilon}\right] \otimes N^{\vee}, 1_{n}\right) \simeq 0 .
$$


Finally, we observe that $\operatorname{Hom}^{*}\left(\left[Q_{\epsilon}\right],\left[Q_{\nu}\right]\right)=\operatorname{Tot}^{\Pi}(E)$ (see Remark 4.9), where

$$
E=\operatorname{Hom}^{*}\left(\left[Q_{\epsilon}\right],\left[Q_{\nu}\right]^{0}\right) \rightarrow \operatorname{Hom}^{*}\left(\left[Q_{\epsilon}\right],\left[Q_{\nu}\right]^{1}\right) \rightarrow \cdots .
$$

So $\operatorname{Hom}^{*}\left(\left[Q_{\epsilon}\right],\left[Q_{\nu}\right]\right)$ is a convolution of contractible chain complexes and Lemma 4.18 implies the theorem.

Remark 5.14 The above considerations admit marginal generalizations which are not necessary for the proof of the main theorem. For instance, suppose that $A \otimes{ }^{\Pi} B$ denotes the tensor product, $(A \otimes \Pi B)_{n}=\prod_{i+j=n} A_{i} \otimes B_{j}$. Then the argument above shows that $\left[Q_{\nu}\right] \otimes \Pi\left[Q_{\epsilon}\right] \simeq 0$ unless $\epsilon \unlhd \nu$ and the following adjunction holds:

$$
\operatorname{Hom}^{*}(C \otimes A, B) \cong \operatorname{Hom}^{*}\left(C, B \otimes \Pi A^{\vee}\right) \text {. }
$$

These observations lead to the stronger statement below.

Statement Suppose that $A, B \in \operatorname{Kom}(n),\left[Q_{\epsilon}\right] \in\left\langle Q_{\epsilon}\right\rangle$ and $\left[Q_{\nu}\right] \in\left\langle Q_{\nu}\right\rangle$. Then $\epsilon \not \mathcal{v}$ implies that $\operatorname{Hom}^{*}\left(A \otimes\left[Q_{\epsilon}\right], B \otimes\left[Q_{\nu}\right]\right)$ is contractible.

\section{Explicit constructions of resolutions of identity}

In this section the higher-order projectors are constructed for $n=2,3$ and 4 . The general construction can be found in Section 7. Many of the important features of this proof can be seen concretely here when $n=4$. The subscripts used in this section correspond to the sequences introduced in Definition 2.15.

\subsection{Two strands: $P_{(1,-1)}$ and $P_{(1,1)}$}

The second projector $P_{2}$ can be represented by a chain complex of the form

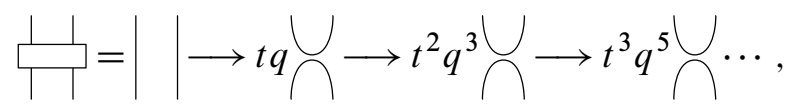

where the first map is a saddle and the last two maps alternate between a difference and a sum of two dots, see [8]. Recalling the box notation from Definition 3.5, we write

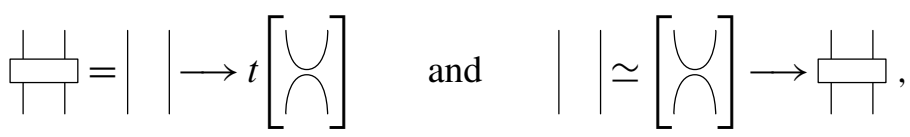

where the map defining the first cone is the saddle appearing in the definition of $P_{2}$, and the map in the second cone is the inclusion of the tail into $P_{2}$. Let us write $t P_{(1,-1)}$ for the subcomplex of $P_{2}$ consisting of terms in homological degree greater than zero 
and set $P_{(1,1)}=P_{2}$. There is a map $i: P_{(1,-1)} \rightarrow P_{(1,1)}$ with $\operatorname{deg}_{t}(i)=1$, which satisfies Cone $(i) \simeq 1_{2}$, where $1_{2}$ is the identity diagram illustrated above.

The chain complex $P_{(1,-1)}$ is idempotent and the map $i$ gives the resolution of identity.

\subsection{Three strands: $P_{(1,-1,1)}, P_{(1,1,-1)}$ and $P_{(1,1,1)}$}

The identity object $1_{3}$ on three strands is given by the union of the identity object on two strands together with an extra strand, $1_{3}=1_{2} \sqcup 1_{1}$. Applying $-\sqcup 1$ to the resolution of identity in the previous section we obtain

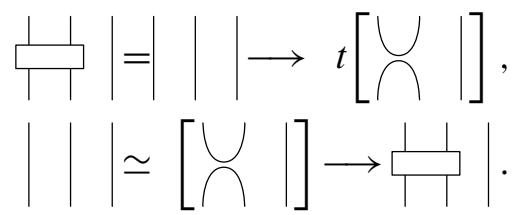

Lemma 3.8 implies that the third universal projector $P_{3}=P_{(1,1,1)}$ can be chosen to be equal to the cone $P_{(1,1)} \sqcup 1 \rightarrow t P_{(1,1,-1)}$. Pictorially,

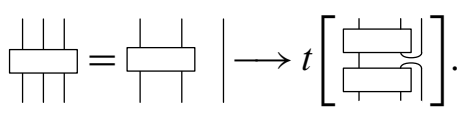

Consider the contractible chain complex Cone $(-\mathrm{Id})=P_{(1,1,-1)} \rightarrow t P_{(1,1,-1)}$. Using the second equation above and from gluing on the contractible chain complex it follows that $1_{3}$ is homotopic to

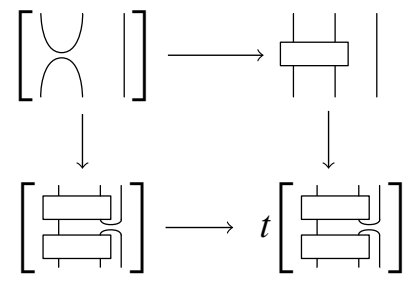

by Lemma 4.24. Using the triangle (6-1) above and reassociating allows us to write this complex in terms of the projectors $P_{(1,-1,1)}=P_{(1,-1)} \sqcup 1, P_{(1,1,-1)}$ and $P_{(1,1,1)}$. The identity object $1_{3}$ is homotopy equivalent to $R_{3}$.
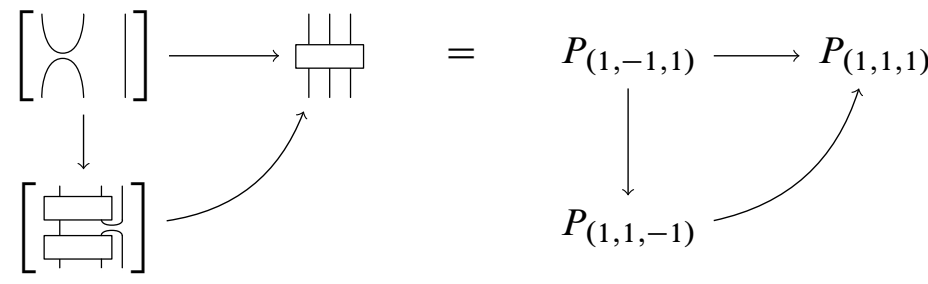
The maps above are compositions of inclusions of tails and differentials from chain complexes of projectors.

\subsection{Four strands}

In the previous section we obtained a resolution of the identity on three strands. The identity object $1_{4}$ on four strands is given by the union of the identity object on three strands together with an extra strand, $1_{4}=1_{3} \sqcup 1_{1}$. Applying $-\sqcup 1$ to the resolution of identity in the previous section we obtain the diagram pictured below:

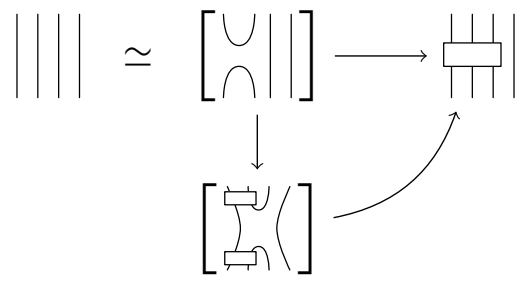

Now Lemma 3.8 implies that the fourth universal projector $P_{4}=P_{(1,1,1,1)}$ can be chosen to be equal to the cone $P_{3} \sqcup 1 \rightarrow t P_{(1,1,1,-1)}$,

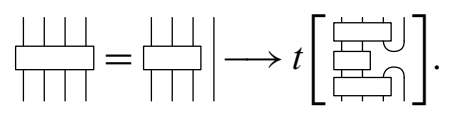

Using Lemma 4.24 we can add the contractible chain complex

$$
\text { Cone }(-\mathrm{Id})=P_{(1,1,1,-1)} \rightarrow t P_{(1,1,1,-1)}
$$

to the decomposition above to obtain a homotopy equivalent complex on the left-hand side below:
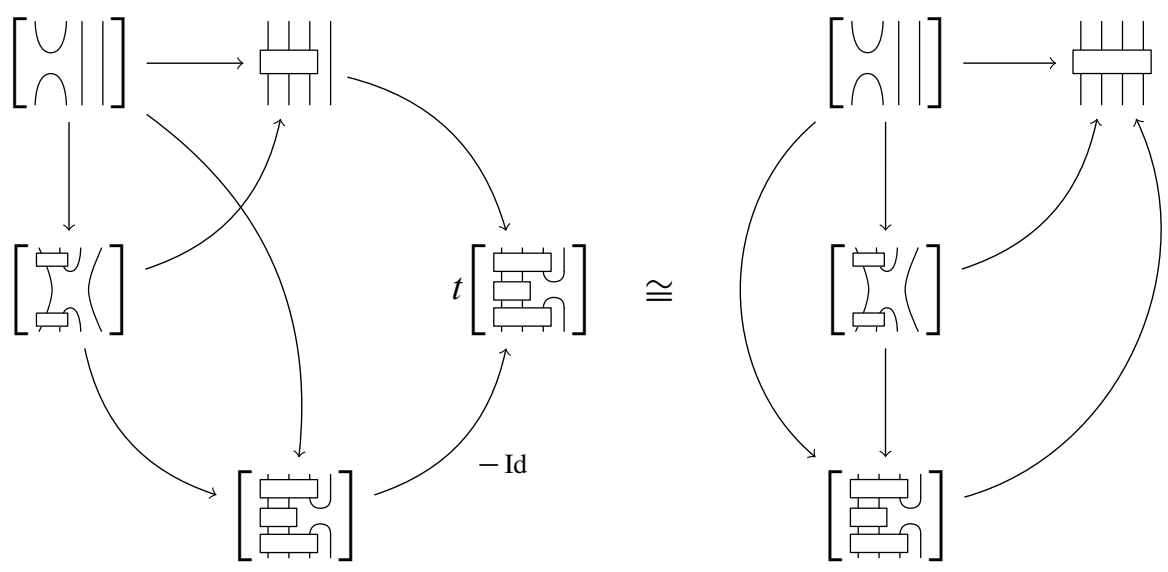
Reassociating allows us to replace $P_{(1,1,1)} \sqcup 1$ in the resolution of identity and yields the isomorphic complex containing the projector $P_{4}$ on the right-hand side above. Unfortunately, we aren't done because our resolution of identity still consists of terms which do not factor through universal projectors. In order to replace the two offending terms, $P_{(1,1,-1)} \sqcup 1$ and $P_{(1,-1,1)} \sqcup 1$, a bit of work remains. The process by which we replace $P_{(1,1,-1)} \sqcup 1$ will illustrate the general strategy.

We can construct the following chain complex:

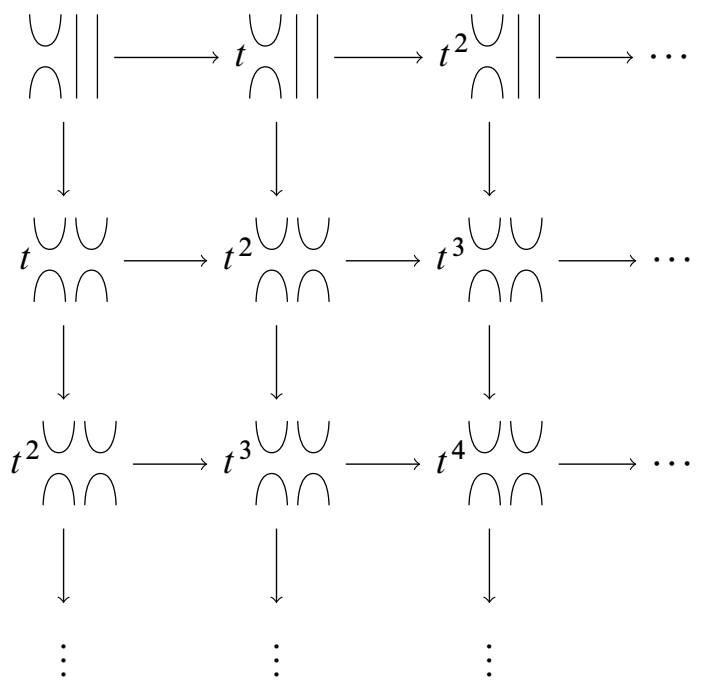

In the more concise bracket notation, this chain complex is

$$
P_{(1,-1,1,1)}=\left[\begin{array}{l}
\cup \biguplus \\
\bigcap \prod
\end{array}\right]
$$

Notice the top row of this bicomplex is $P_{(1,-1,1)} \sqcup 1$; in bracket notation, this is $[\because \|$ ]. The columns of the bicomplex $P_{(1,1,-1,1)}$ are given by $\cup_{\cap}$.

We define $P_{(1,-1,1,-1)}$ to be the tail of the bicomplex $P_{(1,1,-1,1)}$ : the subcomplex consisting of all rows beyond the first (shifted down by 1). In bracket notation, $P_{(1,-1,1,-1)}=[\cup \cup]$. The vertical differential of the bicomplex $P_{(1,1,-1,1)}$ determines a map $\delta: P_{(1,-1,1)} \sqcup 1 \rightarrow P_{(1,-1,1,-1)}$ such that $P_{(1,-1,1,1)}=\operatorname{Cone}(\delta)$. In pictures,

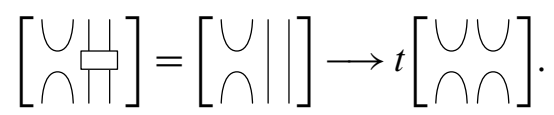

By Lemma 4.24 we add the contractible chain complex Cone $(-\mathrm{Id})=P_{(1,-1,1,-1)} \rightarrow$ $t P_{(1,-1,1,-1)}$ to our decomposition above and reassociate. The identity object $1_{4}$ is 
homotopy equivalent to the left complex pictured below. The complex on the right is obtained by reassociating.

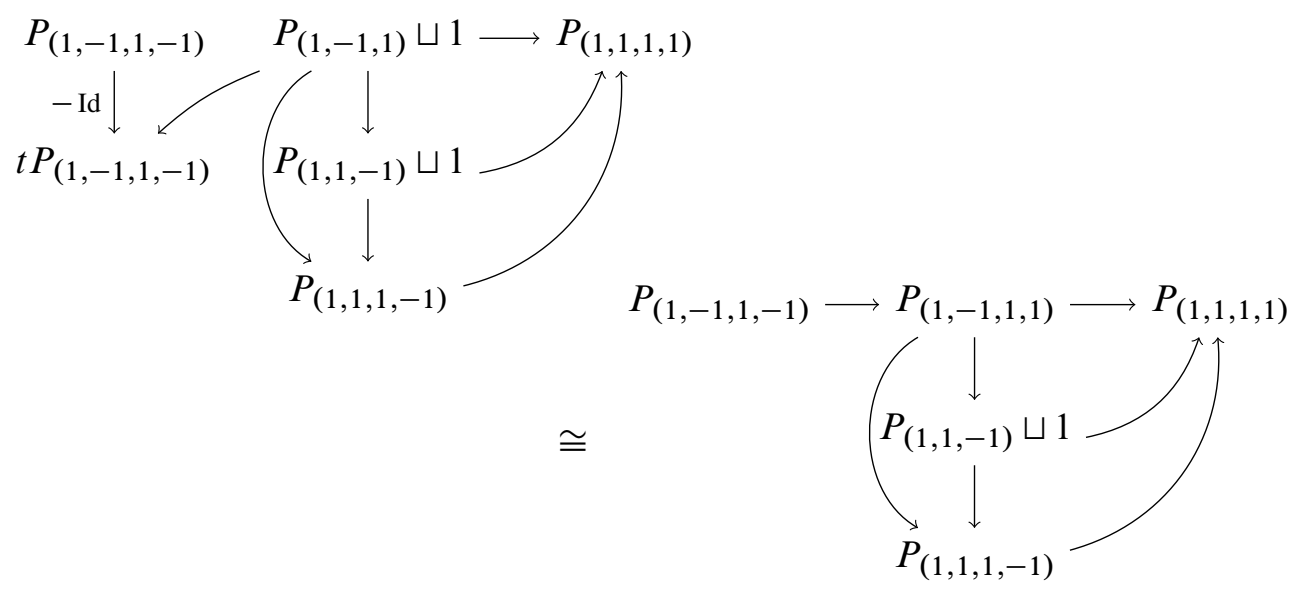

We still have to replace the subcomplex $P_{(1,1,-1)} \sqcup 1$ with a complex that factors through $P_{2}$. In order to accomplish this task we construct a chain complex

$$
P_{(1,1,-1,-1)}=\left[\begin{array}{l}
W \\
\text { तh }
\end{array}\right]
$$

and a chain map $\gamma: P_{(1,1,-1)} \sqcup 1 \rightarrow P_{(1,1,-1,-1)}$ so that

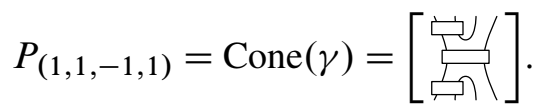

The complex $P_{(1,1,-1)} \sqcup 1=[\stackrel{\vec{f}}{(}(]$ can be written as an iterated cone:

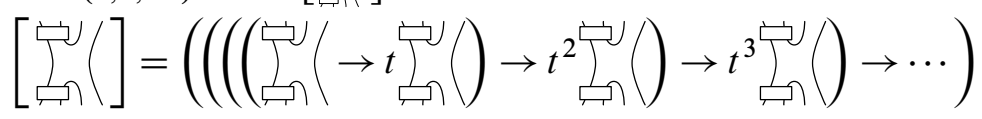

We can also write

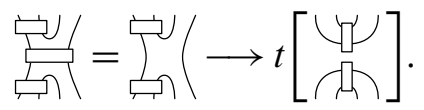

(In the formula above, a degree shift of [2]/[3] has been omitted, following the convention in Definition 3.5.) Using this map we now construct a new triangle of the form

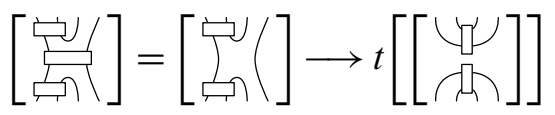

This process is also carried out in Theorem 7.4. We use double brackets above to emphasize that the term on the left is a convolution of convolutions and also to distinguish it from the complex [ [ w w which is the tail of 䆓. 
The first step is to form the cone on the first term of $P_{(1,1,-1)} \sqcup 1$ :

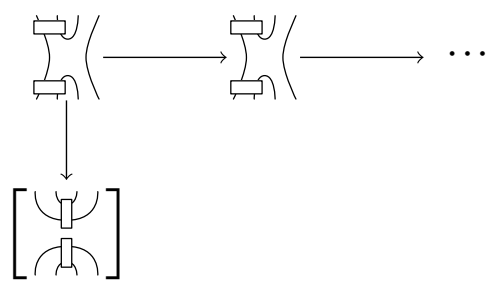

Reassociating shows that the first term in this complex agrees with the desired complex. Now assume by induction that we can form a chain complex in which the first $N$ terms of $P_{(1,1,-1)} \sqcup 1$ have been written in this way:

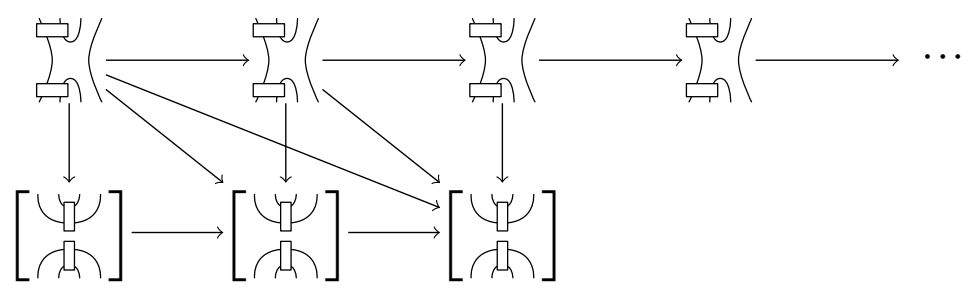

We draw the diagonal arrows to emphasize that the maps in this construction necessarily propagate in a non-trivial way.

After grouping the first $N$ terms of the top and bottom rows within parentheses we consider taking the cone on the $N+1^{\text {st }}$ term:

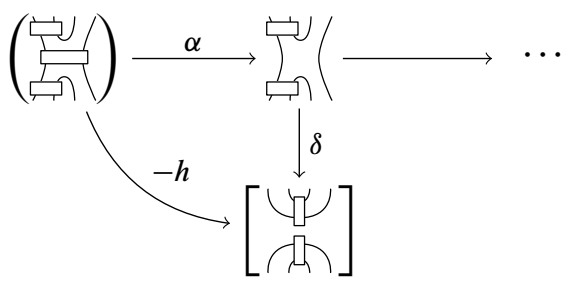

After taking shifts into account, the composition $\delta \circ \alpha$ is a chain map of degree 0 and

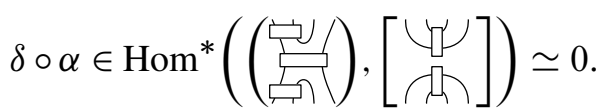

The Hom-complex is contractible by Theorem 5.13. Proposition 4.22 allows us to produce a chain complex with $N+1$ terms of the desired form. This process is stable, adding the $N+1^{\text {st }}$ map does not change any maps which appear earlier, because in Proposition 4.22, the map $\gamma$ is an extension of the map $\beta$. Since there are countably many terms we can use this process to produce the chain complex, $P_{(1,1,-1,1)}$, that we want. 
By construction the top row is $P_{(1,1,-1)} \sqcup 1$ and so we define the bottom row to be $t P_{(1,1,-1,-1)}$. The non-horizontal components of the differential yield a chain map

$$
\eta: P_{(1,1,-1)} \sqcup 1 \rightarrow P_{(1,1,-1,-1)}
$$

such that $P_{(1,1,-1,1)}=\mathrm{Cone}(\eta)$. Our program is resumed by replacing the $P_{(1,1,-1)} \sqcup 1$ term above. By introducing the contractible term

$$
\text { Cone }\left(-\operatorname{Id}_{P_{(1,1,-1,-1)}}\right)=P_{(1,1,-1,-1)} \rightarrow t P_{(1,1,-1,-1)}
$$

to our last complex, we see that $1_{4}$ is homotopy equivalent to the following diagram:

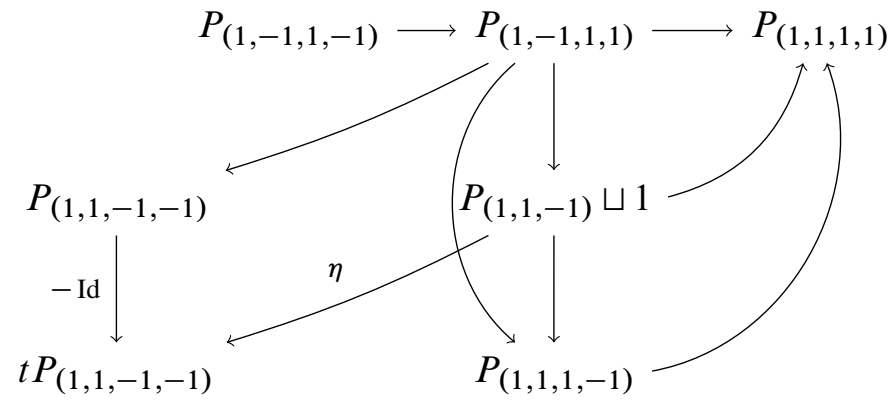

We conclude by reassociating and using the Combing lemma 4.26 to exchange the bad arrow $P_{(1,-1,1,1)} \rightarrow P_{(1,1,-1,-1)}$ with an arrow $P_{(1,-1,1,-1)} \rightarrow P_{(1,1,-1,-1)}$ that respects the dominance order $\unlhd$ on $\mathcal{L}_{4}$ (Definition 2.15). The object $1_{4}$ is homotopic to the following complexes:

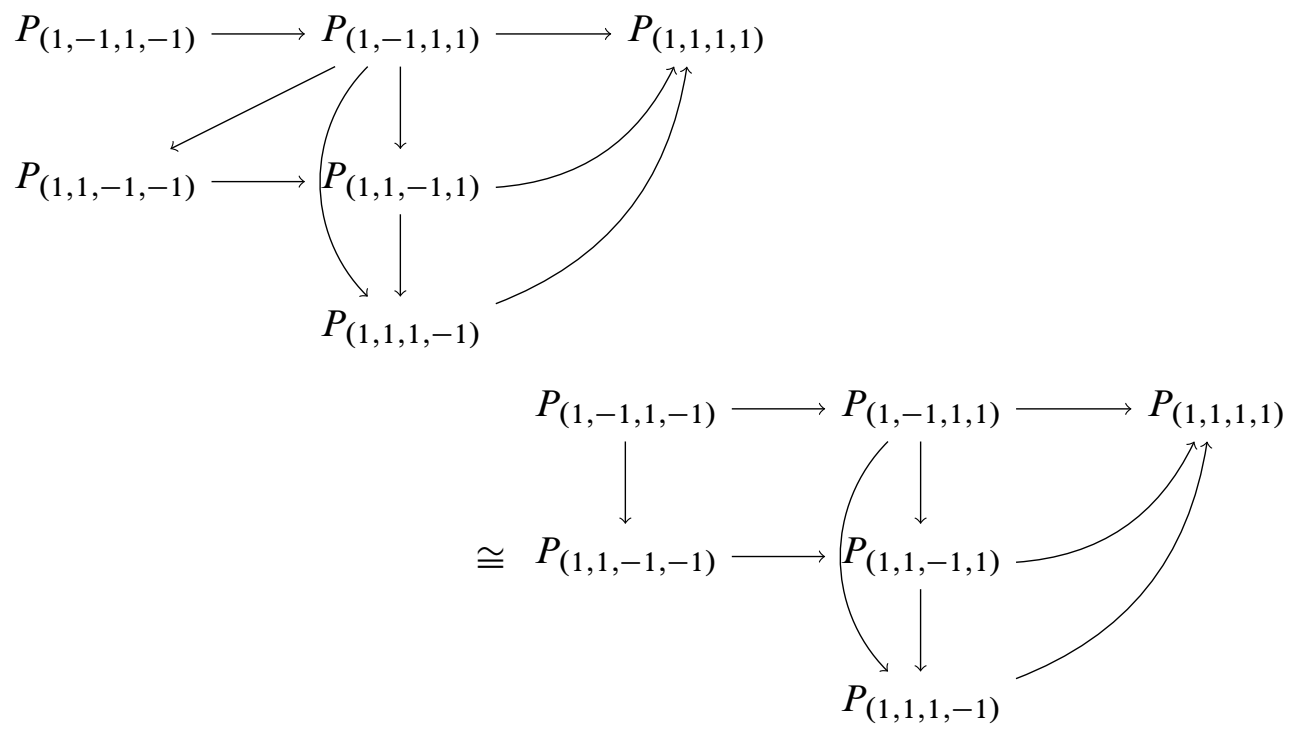


The end result is a resolution of identity on four strands in which all of the terms factor through universal projectors of the form $P_{4-2 k}$ for $k=0,1,2$ and all maps between terms respect the dominance order.

Vista 6.4 In order to accomplish our task, we needed two basic maneuvers. The first was gluing a contractible chain complex onto our resolution without changing the homotopy type using Lemma 4.24. The second was the construction of chain complexes suitable for substitution. The first step is provided by Lemma 3.8, while Proposition 4.22 and Theorem 5.13 allow us to construct more sophisticated sorts of substitutions.

In order to construct the $P_{\epsilon}$, a general version of the argument given above is carried out in Section 7. The reader may refer to this section for intuition.

\section{General construction of the resolution of identity}

In this section we categorify the equations

$$
1_{n}=\sum_{\epsilon \in \mathcal{L}_{n}} p_{\epsilon} \quad \text { and } \quad p_{\epsilon} p_{\nu}=\delta_{\epsilon \nu} p_{\epsilon}
$$

of Theorem 2.20. This is accomplished by constructing chain complexes $P_{\epsilon} \in \operatorname{Kom}(n)$ for each sequence $\epsilon \in \mathcal{L}_{n}$ which satisfy idempotence and orthogonality properties, $P_{\epsilon} \otimes P_{\nu} \simeq \delta_{\epsilon \nu} P_{\epsilon}$. In the process of constructing the projectors $P_{\epsilon}$, we build the resolution of identity $R_{n}$; a convolution of projectors $P_{\epsilon}$ which satisfies $1_{n} \simeq R_{n}$. The Euler characteristic $\mathrm{K}_{0}\left(1_{n}\right)=\mathrm{K}_{0}\left(R_{n}\right)$ can be identified with the first equation above.

In Theorem 5.13, we showed that $\epsilon \not \mathcal{v}$ implies $\operatorname{Hom}^{*}\left(\left[Q_{\epsilon}\right],\left[Q_{\nu}\right]\right) \simeq 0$, for each pair of convolutions $\left[Q_{\epsilon}\right] \in\left\langle Q_{\epsilon}\right\rangle$ and $\left[Q_{\nu}\right] \in\left\langle Q_{\nu}\right\rangle$. The theorem below exploits this fact in order to build triangles relating convolutions in the hulls $\left\langle Q_{\epsilon \cdot(+1)}\right\rangle,\left\langle Q_{\epsilon \cdot(-1)}\right\rangle$ and $\left\langle Q_{\epsilon} \sqcup 1\right\rangle$. An immediate consequence is Corollary 7.2, which constructs chain complexes $P_{\epsilon} \in\left\langle Q_{\epsilon}\right\rangle$. These chain complexes categorify the idempotents $p_{\epsilon} \in \mathrm{TL}_{n}$.

Theorem 7.1 For each sequence $\epsilon \in \mathcal{L}_{n}$ and convolution $\left[Q_{\epsilon}\right] \in\left\langle Q_{\epsilon}\right\rangle$, there exists a convolution $\left[Q_{\epsilon \cdot(-1)}\right] \in\left\langle Q_{\epsilon \cdot(-1)}\right\rangle$ and a chain map

$$
\delta:\left[Q_{\epsilon}\right] \sqcup 1 \rightarrow\left[Q_{\epsilon \cdot(-1)}\right]
$$

of homological and internal degree zero such that

$$
\operatorname{Cone}(\delta) \in\left\langle Q_{\epsilon \cdot(+1)}\right\rangle \text {. }
$$


The proof below is a generalization of the obstruction-theoretic argument used to construct the map $\eta: P_{(1,1,-1)} \sqcup 1 \rightarrow t P_{(1,1,-1,-1)}$ in Section 6.3. The convolution $\left[Q_{\epsilon \cdot(+1)}\right]$ will be defined as $\operatorname{Cone}(\delta)$.

Proof Let $S \subset\left\langle Q_{\epsilon}\right\rangle$ denote the collection of chain complexes for which the theorem is true. In order to prove the theorem we show that $Q_{\epsilon} \in S$ and that $S$ is closed under convolution. These two statements imply that $\left\langle Q_{\epsilon}\right\rangle \subset S$.

In order to show that $Q_{\epsilon} \in S$ we must chase our own definitions. By definition, there is a chain complex $A_{\epsilon} \in \mathrm{Kom}^{*}(n)$ such that $Q_{\epsilon}=A_{\epsilon} \otimes P_{k} \otimes \bar{A}_{\epsilon}$ where $|\epsilon|=k$. By Lemma 3.8, there is a triangle $P_{k+1}=P_{k} \sqcup 1 \stackrel{\delta^{\prime}}{\rightarrow} t T$ where $T$ denotes the tail of the projector $P_{k+1}$. Setting $\left[Q_{\epsilon \cdot(-1)}\right]=\left(A_{\epsilon} \sqcup 1\right) \otimes T \otimes\left(\bar{A}_{\epsilon} \sqcup 1\right)$ and $\delta=\mathrm{Id} \otimes \delta^{\prime} \otimes \mathrm{Id}$ shows that $Q_{\epsilon} \in S$.

The remainder of the proof shows that $S$ is closed under convolutions. Suppose that $\left[Q_{\epsilon}\right] \in\left\langle Q_{\epsilon}\right\rangle$ is a convolution. So $\left[Q_{\epsilon}\right]=\operatorname{Tot}(E)$ where $E=\left\{\left(E_{i}\right), q_{i j}\right\}$ and the $E_{i} \in\left\langle Q_{\epsilon}\right\rangle$ are chain complexes for which the theorem holds. By assumption there are chain complexes $T_{i} \in\left\langle Q_{\epsilon \cdot(-1)}\right\rangle$ and maps

$$
\delta_{i}: E_{i} \sqcup 1 \rightarrow T_{i} \quad \text { such that } \operatorname{Cone}\left(\delta_{i}\right) \in\left\langle Q_{\epsilon \cdot(+1)}\right\rangle .
$$

We wish to define a chain complex $\left[Q_{\epsilon \cdot(+1)}\right] \in\left\langle Q_{\epsilon \cdot(+1)}\right\rangle$ which, as a graded object, is a sum of the complexes appearing in the diagram below:

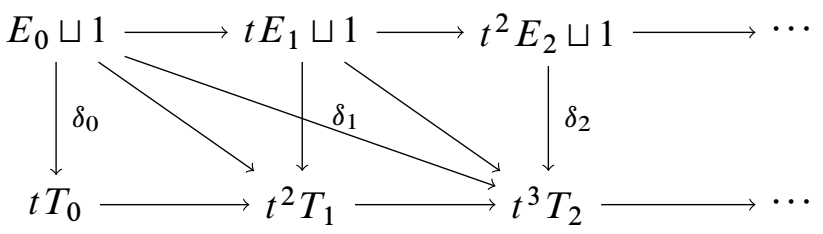

The convolution $\left[Q_{\epsilon \cdot(+1)}\right]$ will be defined as a direct limit of truncations $\left[Q_{\epsilon \cdot(+1)}\right]_{[0, r]}$, see Definition 4.16. We proceed by induction on $r$.

If $r=0$ then set $\left[Q_{\epsilon \cdot(+1)}\right]_{[0,0]}=\operatorname{Cone}\left(\delta_{0}\right)$. Assume that $\left[Q_{\epsilon \cdot(+1)}\right]_{[0, r]}$ has constructed and let $\left[Q_{\epsilon} \sqcup 1\right]_{[0, r]}$ denote the corresponding truncation of $\left[Q_{\epsilon}\right] \sqcup 1$, which appears as the top row of $\left[Q_{\epsilon \cdot(+1)}\right]_{[0, r]}$. The differential on $\left[Q_{\epsilon}\right] \sqcup 1$ gives a chain map $\alpha:\left[Q_{\epsilon} \sqcup 1\right]_{[0, r]} \rightarrow t^{r} E_{r+1} \sqcup 1$. Let $z$ be the composition of the maps in the diagram

$$
\left[Q_{\epsilon \cdot(+1)}\right] \stackrel{\pi}{\longrightarrow}\left[Q_{\epsilon} \sqcup 1\right]_{[0, r]} \stackrel{\alpha}{\longrightarrow} t^{r} E_{r+1} \stackrel{(-1)^{r} \delta_{r+1}}{\longrightarrow} t^{r} T_{r+1},
$$

where $\pi$ is the projection of $\left[Q_{\epsilon \cdot(+1)}\right]_{[0, r]}$ onto its top row. By Theorem 5.13 the map $z$ belongs to a contractible Hom-space,

$$
z \in \operatorname{Hom}^{*}\left(\left[Q_{\epsilon \cdot(+1)}\right], t^{r} T_{r+1}\right) \simeq 0 .
$$


Therefore, $z=d(h)$ is a boundary and Proposition 4.22 allows us to produce a chain complex with $r+1$ terms of the desired form.

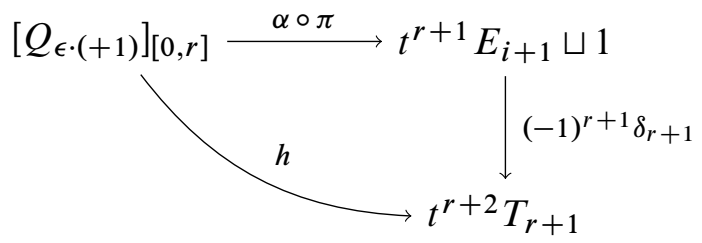

Observe that the differentials only point south or east. The new column in this complex is $t^{r+1} \operatorname{Cone}\left(\delta_{r+1}\right)$. Since $\pi$ is the identity on the top row and zero on the bottom row, the top row of this complex is the corresponding truncation of $\left[Q_{\epsilon}\right] \sqcup 1$. This construction is stable for the same reasons as in Section 6.3.

Defining $\left[Q_{\epsilon \cdot(+1)}\right]$ to be the limit of the resulting directed system completes the proof.

Corollary 7.2 For each sequence $\epsilon \in \mathcal{L}_{n}$, there exist a chain complex $P_{\epsilon} \in\left\langle Q_{\epsilon}\right\rangle$ in the hull of $Q_{\epsilon}$ and maps

$$
\delta_{\epsilon}: P_{\epsilon} \sqcup 1 \rightarrow t P_{\epsilon \cdot(-1)}
$$

such that $P_{\epsilon \cdot(+1)}=\operatorname{Cone}\left(\delta_{\epsilon}\right)$.

The corollary below follows from the argument given above.

Corollary 7.3 For each $n>0$ and each sequence $\epsilon \in \mathcal{L}_{n}$, the triangle

$$
P_{\epsilon} \sqcup 1=P_{\epsilon \cdot(-1)} \rightarrow P_{\epsilon \cdot(+1)}
$$

descends to Equation (2-8) of Section 2.14 in the Grothendieck group $\mathrm{K}_{0}(\operatorname{Kom}(n))$.

The following theorem is a generalization of the resolution of identity found in Section 6.3. This is the main result of this section.

Theorem 7.4 For each $n>0$, there is a twisted complex $R_{n}=\left\{\left(P_{\epsilon}\right), d_{\epsilon \nu}\right\}_{\epsilon \in \mathcal{L}_{n}} \in$ $\operatorname{Tw} \operatorname{Kom}(n)$ such that

$$
1_{n} \simeq R_{n}
$$

Proof When $n=1$ we set $R_{1}=1$. Assume by induction that there is a twisted complex $R_{n-1}=\left\{\left(P_{\epsilon}\right), d_{\epsilon \nu}\right\}_{\epsilon \in \mathcal{L}_{n-1}}$ such that $1_{n-1} \simeq R_{n-1}$. Placing a disjoint strand next to everything yields

$$
1_{n}=1_{n-1} \sqcup 1=R_{n-1} \sqcup 1=\left\{\left(P_{\epsilon} \sqcup 1\right), d_{\epsilon v} \sqcup 1\right\} .
$$


Corollary 4.25 and Corollary 7.2 imply that we can replace each $P_{\epsilon} \sqcup 1$ with $P_{\epsilon \cdot(-1)} \rightarrow$ $P_{\epsilon \cdot(+1)}$ obtaining an equivalence

$$
1_{n} \simeq\left\{P_{\epsilon \cdot(-1)} \stackrel{f_{\epsilon}}{\longrightarrow} P_{\epsilon \cdot(+1)},\left(\begin{array}{cc}
d_{P_{\epsilon \cdot(-1)}} & 0 \\
f_{\epsilon} & d_{P_{\epsilon \cdot(+1)}}
\end{array}\right)\right\} \cong\left\{P_{\epsilon \cdot( \pm 1)}, d_{\epsilon \nu}^{\prime}\right\} .
$$

The right-hand side is a twisted complex indexed by elements of $\mathcal{L}_{n}$. There may be maps which do not respect the dominance order $\unlhd$ on $\mathcal{L}_{n}$. However, when $\epsilon \unlhd v$ the Hom-space from $P_{\epsilon}$ to $P_{v}$ is contractible:

$$
\operatorname{Hom}^{*}\left(P_{\epsilon}, P_{\nu}\right) \simeq 0 \text {. }
$$

Applications of the Combing lemma 4.26 allow us to exchange maps in $\left\{P_{\epsilon \cdot( \pm 1)}, d_{\epsilon \nu}^{\prime}\right\}$ which do not respect the dominance order for those that do. The resulting twisted complex is the resolution of identity $R_{n}$.

Remark 7.5 When referring to a chain complex in $R_{n} \in \operatorname{Kom}(n)$ the resolution of identity $R_{n}$ is defined to be the convolution $\operatorname{Tot}\left(R_{n}\right)$, see Definition 4.6.

Remark 7.6 In the Grothendieck group $\mathrm{K}_{0}(\operatorname{Kom}(n))$, the resolution of identity becomes the equation $1_{n}=\sum_{\epsilon \in \mathcal{L}_{n}} p_{\epsilon}$ from Section 2.14. From the discussion in Section 2.10, we see that $R_{n}$ categorifies the decomposition of $V_{1}^{\otimes n}$ into irreducible representations.

Remark 7.7 In the decategorified setting, representations decompose into direct sums of irreducible representations. After categorification we have learned that this decomposition is maintained up to homotopy, but the irreducible components now have non-trivial maps between them.

\section{Higher-order projectors}

In this section we will define the universal higher-order projectors and articulate the sense in which the resolutions of identity $R_{n}$ produced in Sections 6 and 7 yield categorifications of the idempotents $P_{n, k}$ defined in Section 2.10. While the axioms of Definition 8.4 given below are sufficient to characterize the projectors $P_{n, k}$ uniquely up to homotopy, we will see that the $P_{n, k}$ also satisfy a number of other useful properties analogous to those enumerated in Section 2.14 . We begin by introducing a few definitions similar to those of Section 2.1.

Just as elements $a \in \operatorname{TL}(n, m)$ have a notion of through-degree (Definition 2.6), chain complexes $A \in \operatorname{Kom}(n, m)$ have a corresponding notion of through-degree. 
Definition 8.1 Suppose that $A \in \operatorname{Cob}(n, m)$ is a Temperley-Lieb diagram. Then $A$ factors as a composition $A=C \otimes B$, where

$$
B \times C \in \operatorname{Cob}(n, l) \times \operatorname{Cob}(l, m) .
$$

The through-degree $\tau(A)$ of $A$ is equal to the minimal $l$ in such a factorization. If $A \in \operatorname{Kom}(n, m)$ is a chain complex of Temperley-Lieb diagrams $\left\{A_{i}\right\}$, then $\tau(A)=$ $\max _{i} \tau\left(A_{i}\right)$.

We now define subcategories $\operatorname{Kom}^{k}(n)$ of $\operatorname{Kom}(n)$. Elements of $\operatorname{Kom}^{k}(n)$ will be convolutions of complexes which factor through the universal projector $P_{k}$. If $A \in$ $\operatorname{Kom}^{k}(n)$ then $\tau(A)=k$. Compare to Remark 2.8.

Definition 8.2 A chain complex $C \in \operatorname{Kom}(n, m)$ factors through $P_{k}$ when there are objects $A \in \operatorname{Kom}(n, k)$ and $B \in \operatorname{Kom}(k, m)$ such that

$$
C \cong A \otimes P_{k} \otimes B
$$

Let $\operatorname{Kom}^{k}(n, m) \subset \operatorname{Kom}(n, m)$ be the full subcategory of convolutions of chain complexes which factor through $P_{k}$. We have analogous notions of subcategories $\operatorname{Kom}^{*, k}(n, m)$ and $\operatorname{Tw}_{K_{o m}}^{k}(n, m)$ in $\operatorname{Kom}^{*}(n, m)$ and $\operatorname{Tw} \operatorname{Kom}(n, m)$ respectively. See Section 4.1 for definitions of these categories.

The next lemma tells us that chain complexes which factor through various universal projectors $P_{k}$ compose in a predictable manner.

Lemma 8.3 For each $A \in \operatorname{Kom}^{k}(n, m)$ and $B \in \operatorname{Kom}^{l}(m, r)$, if $k \neq l$ then

$$
B \otimes A \simeq 0 .
$$

Proof Observe that composing complexes which factor through projectors $P_{k}$ and $P_{l}$ with $k \neq l$ produces a complex containing a turnback; this is contractible by Theorem 3.7. The composite twisted complex lies in the hull of a collection of contractible complexes and therefore it is contractible by Lemma 4.18.

We will now state what is meant by universal higher-order projectors.

Definition 8.4 A chain complex $P \in \operatorname{Kom}(n)$ is a $k^{\text {th }}$ universal higher-order projector if:

(1) The through-degree $\tau(P)$ of $P$ is equal to $k$. 
(2) $\quad P$ vanishes when the number of turnbacks is sufficiently high. For each $l \in \mathbb{Z}_{+}$ and $a \in \operatorname{Cob}(n, l)$ if $\tau(a)<k$ then

$$
a \otimes P \simeq 0 \text { and } P \otimes \bar{a} \simeq 0 .
$$

(3) There exists a chain complex $C \in \operatorname{Kom}(n)$ with $\tau(C)<k$ and a twisted complex

$$
D=1_{n} \rightarrow C \rightarrow t P
$$

such that

$$
a \otimes D \simeq 0 \quad \text { and } \quad D \otimes \bar{a} \simeq 0
$$

for all diagrams $a \in \operatorname{Cob}(n, m)$ such that $\tau(a) \leq k$.

For each sequence $\epsilon \in \mathcal{L}_{n}$, there is a complex $Q_{\epsilon}$ (see Definition 5.9) and if $|\epsilon|=k$ then $Q_{\epsilon}$ factors through $P_{k}$ by construction. It follows that each object $A \in\left\langle Q_{\epsilon}\right\rangle$ must factor through $P_{k}$. In particular, $P_{\epsilon} \in \operatorname{Kom}^{|\epsilon|}(n)$ for each $\epsilon \in \mathcal{L}_{n}$.

As in Definition 2.23, the constituents of the higher-order projector $P_{n, k}$ consist of projectors $P_{\epsilon}$ with $\epsilon \in \mathcal{L}_{n, k}$. The categorical construction differs in that there are now non-trivial maps between the components, $P_{\epsilon}$. We extract $P_{n, k}$ from the resolution of identity in the definition below.

Definition 8.5 A $k^{\text {th }}$ universal higher-order projector $P_{n, k}$ is the convolution of the subcomplex formed by isotypic components in the resolution of identity,

$$
P_{n, k}=\left(\bigoplus_{\epsilon \in \mathcal{L}_{n, k}} P_{\epsilon}, d_{\epsilon v}\right) \text {. }
$$

Remark 8.6 The projector $P_{n, k}$ is homotopy equivalent to the cone of the inclusion map between relative truncations of $R_{n}$; see Equation (8-1).

Theorem 8.7 The chain complexes $P_{n, k}$ of Definition 8.5 are universal higher-order projectors.

Before proving the theorem we record several observations. By construction $P_{n, k}$ is contained in the hull of the set $\mathcal{Q}=\left\{Q_{\epsilon}: \epsilon \in \mathcal{L}_{n, k}\right\}$. The next observation follows from the discussion preceding the definition.

Observation 8.8 The $k^{\text {th }}$ universal higher-order projector factors through the universal projector $P_{k}$. In particular, $P_{n, k} \in \operatorname{Kom}^{k}(n)$. 
Since each $P_{n, k}$ is the restriction of the resolution of identity to the subcomplex consisting of the $P_{\epsilon}$ with $|\epsilon|=k$ we can write a resolution of identity purely in terms of the higher-order projectors.

Observation 8.9 We have

$$
1_{n} \simeq P_{n, n(\bmod 2)} \rightarrow \cdots \rightarrow P_{n, n-4} \rightarrow P_{n, n-2} \rightarrow P_{n, n}
$$

The diagram on the right usually contains higher differentials, $P_{n, i} \rightarrow P_{n, j}$, when $i<j$.

We are ready to prove that the chain complexes $P_{n, k}$ extracted from the resolution of identity above satisfy the properties listed in Definition 8.4.

Proof of Theorem 8.7 The first property, $\tau\left(P_{n, k}\right)=k$, follows from Observation 8.8 above.

Suppose that $a \in \operatorname{Cob}(n, l)$ is a diagram with $\tau(a)<k$. Again, by Observation 8.8 the complex $a \otimes P_{n, k}$ is contained in the hull of $\left\{a \otimes Q_{\epsilon}:|\epsilon|=k\right\}$, but $a \otimes Q_{\epsilon} \simeq 0$ because $Q_{\epsilon}$ factors through $P_{k}$. Each complex in the hull of a collection of contractible complexes is contractible by Lemma 4.18 .

Rotating distinguished triangles in Observation 8.9 above gives the homotopy equivalence

$$
1_{n} \rightarrow t\left(P_{n, n(\bmod 2)} \rightarrow \cdots \rightarrow P_{n, k-2}\right) \rightarrow P_{n, k} \simeq P_{n, k+2} \rightarrow \cdots \rightarrow P_{n, n} .
$$

Let $D$ be the left-hand side of this equation and set $C$ to be the middle term

$$
C=t\left(P_{n, n(\bmod 2)} \rightarrow \cdots \rightarrow P_{n, k-2}\right),
$$

so that the third property follows.

We have seen that the chain complexes $P_{n, k}$ defined above are $k^{\text {th }}$ universal higherorder projectors. The next theorem states that each chain complex $P$ satisfying the properties of Definition 8.4 is homotopy equivalent to the chain complex $P_{n, k}$.

Theorem 8.10 If $P \in \operatorname{Kom}(n)$ is a $k^{\text {th }}$ universal higher-order projector then $P$ is homotopy equivalent to $P_{n, k}$ of Definition 8.4. 
Proof Suppose that $P \in \operatorname{Kom}(n)$ satisfies properties (1)-(3) of Definition 8.4 above. From Observation 8.9 we have the resolution of identity

$$
1_{n} \simeq P_{n, n(\bmod 2)} \rightarrow \cdots \rightarrow P_{n, n-4} \rightarrow P_{n, n-2} \rightarrow P_{n, n} .
$$

Applying $P \otimes-$ above yields $P=P \otimes 1_{n} \simeq P_{n, k} \otimes P$. By property (3) there are complexes $C$ and $D$, where

$$
D=1_{n} \rightarrow C \rightarrow t P .
$$

Since $\tau\left(P_{n, k}\right)=k$, property (3) also implies that $P_{n, k} \otimes D \simeq 0$. Now $P_{n, k} \otimes D \simeq 0$ tells us that

$$
0 \simeq P_{n, k} \otimes 1_{n} \rightarrow P_{n, k} \otimes C \rightarrow t P_{n, k} \otimes P=\operatorname{Cone}\left(P_{n, k} \rightarrow P_{n, k} \otimes P\right)
$$

because $P_{n, k} \otimes C \simeq 0$. However, since Cone $(f) \simeq 0$ if and only if $f$ is a homotopy equivalence, the above equation implies that $P_{n, k} \otimes P \simeq P$ and therefore $P_{n, k} \simeq P$.

Now that existence and uniqueness have been shown, we continue our discussion with a series of observations.

Proposition 8.11 The top projector $P_{n, n}$ is a universal projector $P_{n}$.

Proof This can be seen indirectly by comparing the three properties in Definition 8.4 with those of Theorem 3.7. Alternatively, this can be seen directly by tracing through the construction in either Section 6 or Section 7. See [8] for an extended discussion of the axioms found in Theorem 3.7.

Remark 8.12 The universal projectors $P_{n, n}$ of Theorem 3.7 were first categorified in [8;11;24]. The bottom projectors $P_{2 n, 0} \in \operatorname{Kom}(n)$ were categorified and related to the Hochschild homology of Khovanov's ring $H_{n}$ in [23].

Proposition 8.13 The $P_{n, k}$ are mutually orthogonal idempotents,

$$
P_{n, k} \otimes P_{n, l} \simeq \delta_{k, l} P_{n, k} .
$$

Proof When $k \neq l$ the statement $P_{n, k} \otimes P_{n, l} \simeq 0$ follows from Observation 8.8 and Lemma 8.3 above. If $k=l$ then considering the resolution of identity

$$
1_{n} \simeq P_{n, n(\bmod 2)} \rightarrow \cdots \rightarrow P_{n, n-2} \rightarrow P_{n, n},
$$

composing with $P_{n, k}$ gives $P_{n, k}=P_{n, k} \otimes 1_{n} \simeq P_{n, k} \otimes P_{n, k}$.

The proof of the proposition below is analogous to the proof of Proposition 2.27. 
Proposition 8.14 Suppose that $a \in \operatorname{Kom}(m, n)$. Then

$$
a \otimes P_{m, k} \simeq P_{n, k} \otimes a .
$$

In pictures,

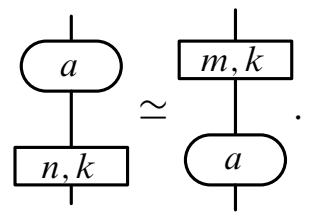

Theorem 5.13 implies that Hom-spaces between convolutions of $Q_{\epsilon}$ and $Q_{\nu}$ are contractible when $\epsilon \not \Perp v$. Since the complexes $P_{\epsilon}$ are convolutions of $Q_{\epsilon}$ and $P_{n, k}$ is constructed using $P_{\epsilon}$ with $\epsilon \in \mathcal{L}_{n, k}$,

$$
\operatorname{Hom}^{*}\left(P_{n, i}, P_{n, j}\right) \simeq 0 \text { when } j<i \text {. }
$$

Theorem 4.20 implies that the differential graded algebra $E=\bigoplus_{i \leq j} \operatorname{Hom}^{*}\left(P_{n, i}, P_{n, j}\right)$ is of fundamental importance. This algebra is a generalization of the endomorphism algebras $\operatorname{End}^{*}\left(P_{n}\right)$. The precise nature of the algebra $\operatorname{End}^{*}\left(P_{n}\right)$ has been the focus of a series of conjectures by Gorsky, Oblomkov, Rasmussen and Shende; see [12].

\subsection{Postnikov decompositions}

In this section we discuss a more algebraic characterization of the projectors $P_{n, k}$ and the resolution of identity $R_{n}$.

Definition 8.16 The resolution of identity $R_{n}$ can be written as a convolution of higher order projectors

$$
1_{n} \simeq R_{n}=P_{n, n(\bmod 2)} \rightarrow \cdots \rightarrow P_{n, n-4} \rightarrow P_{n, n-2} \rightarrow P_{n, n} ;
$$

see Observation 8.9. Truncating the diagram yields triangles of inhomogeneous idempotents

$$
R_{n}=W_{n, k} \rightarrow Z_{n, k},
$$

where

$W_{n, k}=P_{n, n(\bmod n)} \rightarrow \cdots \rightarrow P_{n, k-2} \quad$ and $\quad Z_{n, k}=P_{n, k} \rightarrow P_{n, k+2} \rightarrow \cdots \rightarrow P_{n, n}$.

For each $k$, there is a canonical inclusion $Z_{n, k} \rightarrow Z_{n, k-2}$, which yields a triangle

$$
P_{n, k-2} \rightarrow Z_{n, k} \rightarrow Z_{n, k-2} .
$$


It follows from the triangles in Equation (8-1) that the inhomogeneous idempotents $Z_{n, k}$ determine the higher-order projectors $P_{n, k}$ up to homotopy. We will show that the $Z_{n, k}$ can be characterized by localization.

Remark 8.17 For any object $S$, these triangles can be sewn together to obtain the canonical decomposition pictured below:

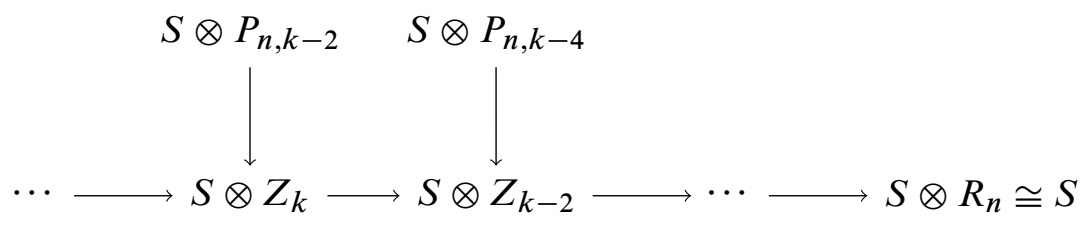

The picture above is a Postnikov-type decomposition. It is well known that such decompositions can be constructed functorially using the language of quotients and localization. In Proposition 8.22 we will show that the chain complex $Z_{n, k}$ determines the Bousfield colocalization with respect to the $k^{\text {th }}$ layer of the through-degree filtration. We must extend the category $\operatorname{Kom}(n)$ so that certain limits are guaranteed to exist.

Definition 8.18 $\left(\mathrm{Ho}_{+}(n)\right)$ Let $\operatorname{Kom}_{+}(n, m)=\operatorname{Kom}_{+}(\operatorname{Cob}(n, m))$ be the closure of $\operatorname{Kom}(n, m)$ under small coproducts. We will use the abbreviations

$$
\operatorname{Kom}_{+}(n)=\operatorname{Kom}_{+}(n, n) \quad \text { and } \quad \operatorname{Ho}_{+}(n)=\operatorname{Ho}\left(\operatorname{Kom}_{+}(n)\right) .
$$

The next definition should be compared to Remark 2.8 .

Definition 8.19 The category $\mathrm{Ho}_{+}(n)$ is filtered by the through-degree $\tau$ defined in Definition 8.1. If $\operatorname{Kom}_{+}^{k}(n) \subset \operatorname{Kom}_{+}(n)$ is the full subcategory consisting of chain complexes of diagrams with through-degree less than $k$, then by setting

$$
\mathrm{Ho}_{+}^{k}(n)=\operatorname{Kom}_{+}^{k}(n)
$$

we obtain the filtration

$$
\cdots \subset \mathrm{Ho}_{+}^{k-1}(n) \subset \mathrm{Ho}_{+}^{k}(n) \subset \mathrm{Ho}_{+}^{k+1}(n) \subset \cdots \quad \text { and } \quad \mathrm{Ho}_{+}(n)=\bigcup_{k} \mathrm{Ho}_{+}^{k}(n) .
$$

The resolution of identity $R_{n}$ and the projectors $P_{n, k}$ exist in $\operatorname{Kom}_{+}(n)$ and the inhomogeneous projector $Z_{n, k}$ induces a functor

$$
-\otimes Z_{n, k}: \mathrm{Ho}_{+}(n) \rightarrow \mathrm{Ho}_{+}(n) .
$$

We would like to show that this functor is the universal functor which annihilates subcategory $\mathrm{Ho}_{+}^{k}(n)$. Before stating the proposition we recall some vocabulary. 
Definition 8.20 Let $\mathcal{T}$ be a triangulated category. Then $L: \mathcal{T} \rightarrow \mathcal{T}$ is a localization functor when $L$ is exact and there is a map $\eta: \operatorname{Id}_{\mathcal{T}} \rightarrow L$ such that $L \eta: L \rightarrow L^{2}$ is invertible and $L \eta=\eta L$. A functor $L: \mathcal{T} \rightarrow \mathcal{T}$ is a colocalization functor when $L^{\mathrm{op}}: \mathcal{T}^{\mathrm{op}} \rightarrow \mathcal{T}^{\mathrm{op}}$ is a localization functor.

Definition 8.21 Let $L: \mathcal{T} \rightarrow \mathcal{T}$ be a colocalization functor. Then an object $X \in \mathcal{T}$ is $L$-acyclic when $L X \cong 0$ and the kernel of $L \operatorname{ker}(L) \subset \mathcal{T}$ is the subcategory formed by all $L$-acyclic objects.

For further detail the reader may consult [20, Chapter 9].

Proposition 8.22 The functor determined by the inhomogeneous projector

$$
-\otimes Z_{n, k}: \mathrm{Ho}^{+}(n) \rightarrow \mathrm{Ho}^{+}(n)
$$

is a colocalization functor and $\operatorname{ker}\left(-\otimes Z_{n, k}\right)=\mathrm{Ho}_{+}^{k}(n)$.

Proof The functor $-\otimes Z_{n, k}$ is exact because it commutes with cones and suspension. If $S \in \mathrm{Ho}_{+}^{k}(n)$ then $S$ is a chain complex consisting of diagrams with through-degree less than $k$ then the second property of Definition 8.4 implies that $S \otimes Z_{n, k} \cong 0$. Conversely, if $S \in \mathrm{Ho}_{+}(n)$ and $S \otimes Z_{n, k} \cong 0$ then

$$
S \cong S \otimes Z_{n, k} \rightarrow S \otimes W_{n, k} \cong S \otimes W_{n, k}
$$

implies that $\tau(S)=\tau\left(S \otimes W_{n, k}\right) \leq \tau\left(W_{n, k}\right)<k$ and therefore $S \in \mathrm{Ho}_{+}^{k}(n)$. We conclude that $\operatorname{ker}\left(-\otimes Z_{n, k}\right)=\mathrm{Ho}_{+}^{k}(n)$.

There is a canonical map $j_{n, k}: Z_{n, k} \rightarrow R_{n}$. If $S \in \mathrm{Ho}_{+}(n)$ then define $\eta_{S}: S \otimes Z_{n, k} \rightarrow$ $S$ by the composition $\varphi_{S} \circ\left(\operatorname{Id}_{S} \otimes j_{n, k}\right)$ where $\varphi_{S}: R_{n} \otimes S \stackrel{\sim}{\longrightarrow} S$. The naturality of the map $\operatorname{Id}_{S} \otimes j_{n, k}$ follows from the definition of monoidal structure. The map $\varphi_{S}$ is natural in $S$ because it can be written as $S \otimes R_{n}=\operatorname{Cone}\left(S \rightarrow C_{S}\right) \stackrel{\sim}{\longrightarrow} S$ where $C_{S}$ is a contractible chain complex. Therefore, $\eta$ is a natural transformation. The properties of $\eta$ follow from idempotence, $Z_{n, k} \otimes Z_{n, k} \cong Z_{n, k}$, and associativity relations.

Acknowledgements The authors would like to thank V Krushkal for his interest. B Cooper was supported in part by the Max Planck Institute for Mathematics in Bonn. 


\section{References}

[1] B Bakalov, A Kirillov, Jr, Lectures on tensor categories and modular functors, University Lecture Series 21, Amer. Math. Soc. (2001) MR1797619

[2] D Bar-Natan, Khovanov's homology for tangles and cobordisms, Geom. Topol. 9 (2005) 1443-1499 MR2174270

[3] J Bernstein, I Frenkel, M Khovanov, A categorification of the Temperley-Lieb algebra and Schur quotients of $U\left(\mathfrak{s l}_{2}\right)$ via projective and Zuckerman functors, Selecta Math. 5 (1999) 199-241 MR1714141

[4] A I Bondal, M M Kapranov, Framed triangulated categories, Mat. Sb. 181 (1990) 669-683 MR1055981 In Russian; translated in Math. USSR-Sb. 70 (1991) 93-107

[5] S Cautis, Clasp technology to knot homology via the affine Grassmannian arXiv: 1207.2074

[6] S Cautis, J Kamnitzer, Knot homology via derived categories of coherent sheaves, I: the sl(2)-case, Duke Math. J. 142 (2008) 511-588 MR2411561

[7] D Clark, S Morrison, K Walker, Fixing the functoriality of Khovanov homology, Geom. Topol. 13 (2009) 1499-1582 MR2496052

[8] B Cooper, V Krushkal, Categorification of the Jones-Wenzl projectors, Quantum Topol. 3 (2012) 139-180 MR2901969

[9] B Cooper, V Krushkal, Handle slides and localizations of categories, Int. Math. Res. Not. 2013 (2013) 2179-2202 MR3061937

[10] P Cvitanović, Group theory: Birdtracks, Lie's, and exceptional groups, Princeton Univ. Press (2008) MR2418111

[11] I Frenkel, C Stroppel, J Sussan, Categorifying fractional Euler characteristics, JonesWenzl projectors and 3j-symbols, Quantum Topol. 3 (2012) 181-253 MR2901970

[12] E Gorsky, A Oblomkov, J Rasmussen, V Shende, Torus knots and the rational DAHA, Duke Math. J. 163 (2014) 2709-2794 MR3273582

[13] S Gukov, M Stošić, Homological algebra of knots and BPS states, from: "Proceedings of the Freedman Fest", (R Kirby, V Krushkal, Z Wang, editors), Geom. Topol. Monogr. 18 (2012) 309-367

[14] M Hogancamp, Morphisms between categorified spin networks arXiv:1209.2732

[15] L H Kauffman, S L Lins, Temperley-Lieb recoupling theory and invariants of 3manifolds, Ann. Math. Studies 134, Princeton Univ. Press (1994) MR1280463

[16] M Khovanov, A categorification of the Jones polynomial, Duke Math. J. 101 (2000) 359-426 MR1740682

[17] M Khovanov, A functor-valued invariant of tangles, Algebr. Geom. Topol. 2 (2002) 665-741 MR1928174 
[18] M Khovanov, Link homology and Frobenius extensions, Fund. Math. 190 (2006) 179-190 MR2232858

[19] M Khovanov, L Rozansky, Matrix factorizations and link homology, Fund. Math. 199 (2008) 1-91 MR2391017

[20] A Neeman, Triangulated categories, Ann. Math. Studies 148, Princeton Univ. Press (2001) MR1812507

[21] N R O'Brian, D Toledo, Y L L Tong, The trace map and characteristic classes for coherent sheaves, Amer. J. Math. 103 (1981) 225-252 MR610475

[22] D Orlov, Derived categories of coherent sheaves and triangulated categories of singularities, from: "Algebra, arithmetic, and geometry: in honor of Yu I Manin, Vol. II", (Y Tschinkel, Y Zarhin, editors), Progr. Math. 270, Birkhäuser, Boston (2009) 503-531 MR2641200

[23] L Rozansky, A categorification of the stable SU(2) Witten-Reshitikhin-Turaev invariant of links in $S^{2} \times S^{1}$ arXiv:1011.1958

[24] L Rozansky, An infinite torus braid yields a categorified Jones-Wenzl projector, Fund. Math. 225 (2014) 305-326 MR3205575

[25] L Rozansky, Khovanov homology of a unicolored B-adequate link has a tail, Quantum Topol. 5 (2014) 541-579 MR3317343

[26] P Seidel, I Smith, A link invariant from the symplectic geometry of nilpotent slices, Duke Math. J. 134 (2006) 453-514 MR2254624

[27] C Stroppel, Parabolic category 0, perverse sheaves on Grassmannians, Springer fibres and Khovanov homology, Compos. Math. 145 (2009) 954-992 MR2521250

[28] B Webster, Knot invariants and higher representation theory, II: The categorification of quantum knot invariants (2013) arXiv:1005.4559v7

[29] H Wenzl, On sequences of projections, C. R. Math. Rep. Acad. Sci. Canada 9 (1987) 5-9 MR873400

[30] H Wu, A colored $\mathfrak{s l}(N)$ homology for links in $S^{3}$, Dissertationes Math. (Rozprawy Mat.) 499 (2014) 1-217 MR3234803

Department of Mathematics, University of Iowa 14 MacLean Hall, Iowa City, 52242-1419, USA

Department of Mathematics, Indiana University Bloomington Rawles Hall, 831 E 3rd St, Bloomington, IN 47405, USA

ben-cooper@uiowa.edu, mhoganca@indiana.edu

Received: 2 December 2013 Revised: 29 August 2014 